\title{
Natural history of SLC11 genes in vertebrates: tales from the fish world
}

\author{
João V Neves ${ }^{1}$, Jonathan M Wilson², Heiner Kuhl ${ }^{3}$, Richard Reinhardt ${ }^{3}$, L Filipe C Castro ${ }^{2}$ and \\ Pedro NS Rodrigues ${ }^{1,4^{*}}$
}

\begin{abstract}
Background: The SLC11A1/Nramp1 and SLC11A2/Nramp2 genes belong to the SLC11/Nramp family of transmembrane divalent metal transporters, with SLC11A1 being associated with resistance to pathogens and SLC11A2 involved in intestinal iron uptake and transferrin-bound iron transport. Both members of the SLC11 gene family have been clearly identified in tetrapods; however SLC11A1 has never been documented in teleost fish and is believed to have been lost in this lineage during early vertebrate evolution. In the present work we characterized the SLC11 genes in teleosts and evaluated if the roles attributed to mammalian SLC11 genes are assured by other fish specific SLC11 gene members.

Results: Two different SLC11 genes were isolated in the European sea bass (Dicentrarchus. labrax), and named s/C11a2- $\alpha$ and slc11a2- $\beta$, since both were found to be evolutionary closer to tetrapods SLC11A2, through phylogenetic analysis and comparative genomics. Induction of s/c11a2- $\alpha$ and s/c11a2- $\beta$ in sea bass, upon iron modulation or exposure to Photobacterium damselae spp. piscicida, was evaluated in in vivo or in vitro experimental models. Overall, slc11a2- $\alpha$ was found to respond only to iron deficiency in the intestine, whereas s/c11a2- $\beta$ was found to respond to iron overload and bacterial infection in several tissues and also in the leukocytes.

Conclusions: Our data suggests that despite the absence of $s / c 11 a 1$, its functions have been undertaken by one of the s/c11a2 duplicated paralogs in teleost fish in a case of synfunctionalization, being involved in both iron metabolism and response to bacterial infection. This study provides, to our knowledge, the first example of this type of sub-functionalization in iron metabolism genes, illustrating how conserving the various functions of the SLC11 gene family is of crucial evolutionary importance.
\end{abstract}

\section{Background}

The solute carrier family 11 ( $S L C 11)$ is a gene family of divalent metal transporters, composed by two functional paralogs, SLC11A1 and SLC11A2. The first member of the SLC11 family, SLC11A1, also known as the natural resistance-associated macrophage protein 1 (NRAMP1), is a divalent cation/proton transporter, which has been proposed to function as either a symporter $[1,2]$ or an antiporter [3,4]. Its expression is almost exclusively restricted to the membrane of late endosomes and lysosomes of immune cells of myeloid lineages (neutrophils, macrophages, dendritic cells) [5,6] and to neuronal cells [7]. SLC11A1 was first found to play a crucial role in the

\footnotetext{
* Correspondence: prodrigu@ibmc.up.pt

'Iron and Innate Immunity, Instituto de Biologia Molecular e Celular (IBMC),

Rua do Campo Alegre 823, 4150-180 Porto, Portugal

Full list of author information is available at the end of the article
}

defense against several unrelated pathogens in mice, such as Mycobacteria, Leishmania and Salmonella [8-10], and several studies have shown that polymorphisms in SLC11A1 are involved in many infectious [11-15] and autoimmune [16-20] diseases in humans. However, the resistance mechanisms attributed to SLC11A1 are still not fully understood $[21,22]$.

The second member of the SLC11 family, SLC11A2, is also referred to as natural resistance-associated macrophage protein 2 (NRAMP2), divalent cation transporter 1 (DCT1) or divalent metal transporter 1 (DMT1). SLC11A2 is a divalent cation/proton symporter [23], with a ubiquitous expression [24-26]. It is known to take up iron from the intestinal brush border in mammals and has been linked to transferrin-dependent iron transport from acidified endosomes to the cytosol in many different tissues $[1,23,25]$. Polymorphisms in SLC11A2 are known to
C Biomed Central

(C) 2011 Neves et al; licensee BioMed Central Ltd. This is an Open Access article distributed under the terms of the Creative Commons Attribution License (http://creativecommons.org/licenses/by/2.0), which permits unrestricted use, distribution, and reproduction in any medium, provided the original work is properly cited. 
underline microcytic anemia in mice and rats, resulting from an impairment of iron recycling and intestinal absorption $[27,28]$.

SLC11 homologs have been found in many distant evolutionarily related groups, such as humans $[29,30]$, mice [9,24], rats [23], birds [31], fishes [32-36], insects [37], nematodes [38], plants [39], yeast [40] and even bacteria [41]. Complete slc11 mRNA coding sequences for teleost fishes have been published in the last few years. Single genes were described in carp (Cyprinus carpio) [42], channel catfish (Ictalarus punctatus) [43], zebrafish (Danio rerio) [44], striped bass (Morone saxatilis) [32], Japanese flounder (Paralichthys olivaceus) [33], turbot (Scophthalmus maximus) [35] and red sea bream (Pagrus major) [34], while two copies have been described in rainbow trout (Oncorhynchus mykiss) [45] and fugu (Takifugu rubripes) [36], with evidence from other teleosts available in various genome databases.

Most animal studies, particularly in teleost fishes, are focused on gene isolation and constitutive expression analysis, with little information on evolutionary and functional aspects. Furthermore, a complex picture emerges from the comparison of phylogenetic and expression studies between fishes and mammals [36]. In fact, little is known about the structure and function of SLC11 in lower vertebrates, although some studies provide evidence for a role of teleost Slc11a2 orthologs in the nutritive metal uptake in the intestine $[46,47]$, and also an involvement in the response to bacterial infection. It has been shown that slc11 mRNA levels are elevated in response to lipopolysaccharide (LPS) and Edwardsiella ictaluri $[43,48]$ in channel catfish, to Vibrio angillarum in turbot [35] and red sea bream [34] and to Mycobacterium in striped bass [32]. However no research has yet elucidated this complex pattern of phylogenetic relationships and functional roles of teleost and mammalian SLC11 genes and no explanations are provided as to why it seems that a homolog of the mammalian SLC11A2 is performing the functions attributed to SLC11A1.

A complement of two SLC11 genes is shared between mammals and teleosts. Whilst it is known that mammalian SLC11A1 and SLC11A2 have likely resulted from genome duplications in early vertebrate ancestry (2R) $[36,49,50]$, the potential role of the teleost fish-specific genome duplication (3R) $[51,52]$ in the evolutionary history of this gene family has not been considered. A comprehensive synteny study could thus help to improve our understanding of the evolution and functional specialization of these genes in teleost fish.

European sea bass (Dicentrarchus labrax) was selected as the teleost model for this study due to the growing amount of data on its immune system [53], the possibility of making use of its partially sequenced genome [54,55] and our previous experience with sea bass models of infection and iron modulation [56-59]. Sea bass is also an important marine aquaculture species in Europe, afflicted by several diseases such as pasteurellosis and vibriosis. Since the early 1980s, its production has risen considerably [60] evolving from extensive culture units to semiintensive or intensive systems. This massive fish concentration leads to an increase in organismal stress and as a consequence fish defenses get compromised, making them more susceptible to pathogen attack. Isolation and characterization of the $s l c 11$ gene(s) in sea bass, as a candidate gene(s) for host defense to infection with pathogens, may be of great benefit to better understand its role in the immune system and to the selection of disease resistant stocks [61]. Moreover, sea bass is part of the Acanthopterygii superorder, which includes stickleback, tetraodon and fugu, organisms that have their genome fully sequenced, making possible a number of comparative genetic studies.

The aims of this study were to identify and characterize the sea bass SLC11 homologs, clarify their evolutionary history and to determine their functional roles, in particular those related with the host iron metabolism and resistance to infection. We evaluated the modulation of SLC11 gene(s) expression in sea bass upon iron modulation (iron deficiency and overload) or exposure to Photobacterium damselae spp. piscicida, in in vivo or in vitro experimental models. We expect that this approach should provide an insight on the evolutionary history of the SLC11 genes in the vertebrata subphylum.

\section{Results}

\section{Southern Blot}

In order to determine the number of copies of slc11 genes in the sea bass genome, a southern blot analysis was performed (Figure 1). After independent digestion of $10 \mu \mathrm{g}$ of genomic DNA with EcoRI or HindII and hybridization with a slc11 DIG-labeled probe, different hybridization bands were visible. No uncut products were observed. Whether digested with EcoRI or HindII, two different hybridization bands were visible, suggesting the existence of two copies of the slc11 genes in the sea bass genome.

\section{Sea bass s/c11 transcripts}

Five different sea bass slc11 transcripts were obtained by primer walking and 5'/3' RACE with liver, spleen and intestine cDNA and analysis of whole-genome shotgun contigs (Max Planck Institute for Molecular Genetics).

One transcript was named slc11a2- $\alpha$ and the other four transcripts were named slc11a2- $\beta 1$ to $\beta 4$, since they were found to share $1557 \mathrm{bp}$ of their coding region (out of a total of $1665 \mathrm{bp}$ for $\beta 1 / \beta 2$ or $1689 \mathrm{bp}$ for $\beta 3$ / $\beta 4)$, with the differences between them limited to the 5 ' and 3' endings (Table 1, Figure 2 and Additional File 1, Figure S1). 


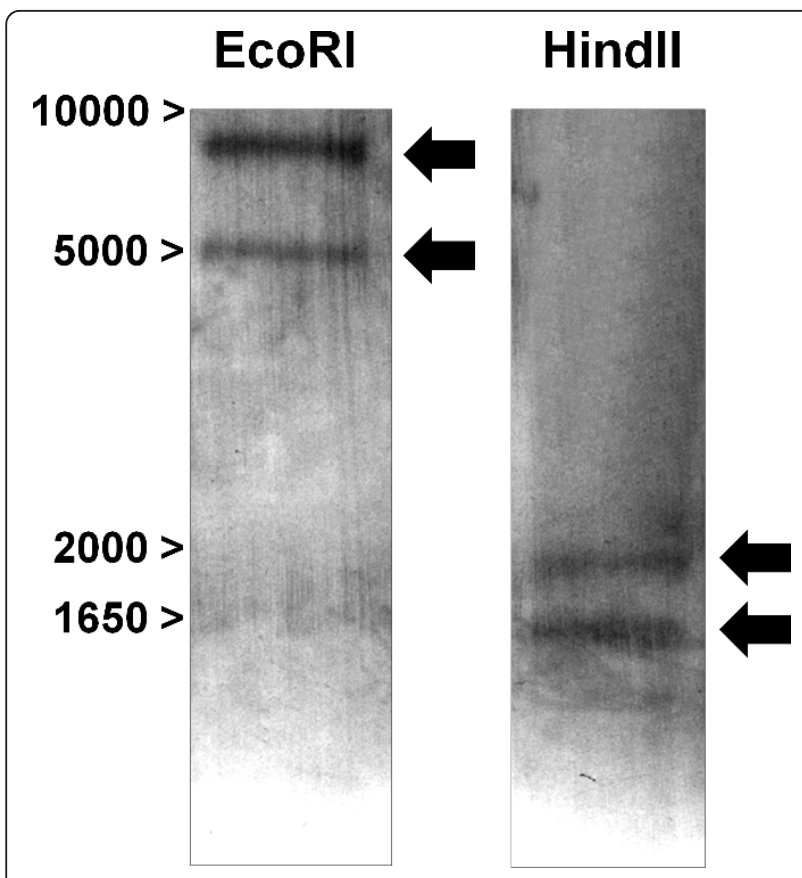

Figure 1 Southern blot analysis. $10 \mu \mathrm{g}$ of genomic DNA were independently digested with EcoRI or Hindll and hybridized with a DIG-labelled s/c11 RNA probe. Molecular weights (bp) are indicated in the left margin.

Slc11a2- $\beta 1$ and slc11a2- $\beta 2$ transcripts are of the same length regarding the coding region, although they differ in the first $34 \mathrm{bp}$, since they result from the alternative usage of either exon 1A ( $\beta 1)$ or exon $1 \mathrm{~B}(\beta 2)$, both with $34 \mathrm{bp}$. They also have in common the final exon (exon 15 ), and the same 3' UTR (Table 1, Figure 2 Additional File 1, Figure S1). Slc11a2- $\beta 3$ and slc11a2- $\beta 4$ transcripts are also of the same length and also differ in the first 34 $\mathrm{bp}$, due to alternative usage of either exon $1 \mathrm{~A}(\beta 3)$ or exon 1B ( $\beta 4)$. Both are slightly larger than slc11a2- $\beta 1$ and slc11a2- $\beta 2$, since exon 15 presents an alternative splice site, losing the final $54 \mathrm{bp}$ (out of $108 \mathrm{bp}$ ) and being partially substituted by the 78 bp of exon 16 (Table 1, Figure 2 and Additional File 1, Figure S1). Slc11a2- $\beta 3$ and slc11a2- $\beta 4$ also share the same 3' UTR, albeit different and smaller than the 3' UTR for slc11a2$\beta 1$ and slc11a2- $\beta 2$ (Table 1, Figure 2 and Additional File 1, Figure S1).

\section{Genomic Organization}

The genomic organization of sea bass slc11a2- $\alpha$ and slc11a2- $\beta$ was analyzed and compared with those of other fishes, amphibians and mammals (see Additional File 2, Figure S2). Exon/intron boundaries were determined by comparison of cDNA, genomic DNA and putative amino acid sequences, splice-site consensus matching and analysis of whole-genome shotgun contigs (Max Planck Institute for Molecular Genetics).

Comparison of genomic DNA sequences with the previously obtained CDNA sequences showed that slc11a2$\alpha$ consists of 15 exons and 14 introns, with a single initiator methionine and stop codon. The genomic interval from the initiator methionine to the stop codon is 7842 bp. Comparison of genomic DNA sequences with the cDNA sequences of the four slc11a2- $\beta$ transcripts showed that those four transcripts result not from four different slc11a2- $\beta$ genes, but rather from the alternative splicing of two 5' exons and two 3' exons of a single slc11a2- $\beta$ gene (as already suggested by the southern blot results), similar to what happens with human SLC11A2 [62,63]. The slc11a2- $\beta$ gene comprises a total of 17 exons, with two initiator methionines and two stop codons. Differences in the 5'-termini are generated by alternative promoters with subsequent, mutually exclusive splicing of the respective first exons to exon 2, whereas differences in the 3'-termini are due to alternative splicing of exon 15, which presents an alternative 5', donor site (corroborated using Alternative Splice Site Predictor $[64,65])$. In two isoforms, this leads to the reading of exon 15 and in the other two isoforms to the partial reading of exon 15 and also exon 16 (Figure 2). The genomic interval from the first initiator methionine in exon $1 \mathrm{~A}$ to the second stop codon in exon 16 is $10543 \mathrm{bp}$.

For both $\alpha$ and $\beta$ genes, exons 2-14 present a high homology with the equivalent exons from other fish and mammals, whereas exons in the $\mathrm{N}$ - and $\mathrm{C}$-terminus are variable in size and sequence. Intron sizes, much like in fugu and tetraodon, are reduced when compared with mammalian homologs. Sea bass slc11a2- $\alpha$ and slc11a2- $\beta$ present a compaction factor of $1.8 \times$ and $1.4 \times$ to human SLC11A1 and $4.6 \times$ and $3.4 \times$ to human SLC11A2, respectively.

Table 1 Sea bass slc11 transcripts

\begin{tabular}{|c|c|c|c|c|c|c|}
\hline Transcript & 5' UTR (bp) & First Exon & Coding (bp) & Last Exon & 3' UTR (bp) & Full lenght (bp) \\
\hline $\operatorname{sict1a2-\alpha }$ & 116 & 1 & 1683 & 15 & 120 & 1919 \\
\hline slct1a2- $\beta 1$ & 203 & $1 \mathrm{~A}$ & 1665 & 15 & 569 & 2437 \\
\hline slctia2- $\beta 2$ & 121 & 1B & 1665 & 15 & 569 & 2355 \\
\hline slc11a2- $\beta 3$ & 203 & $1 \mathrm{~A}$ & 1689 & 16 & 319 & 2211 \\
\hline slc11a2- $\beta 4$ & 121 & $1 \mathrm{~B}$ & 1689 & 16 & 319 & 2129 \\
\hline
\end{tabular}

Sizes of the untranslated regions and coding regions, as well as starting and ending exons for each transcript. 


\section{slc11a2- $\alpha$}

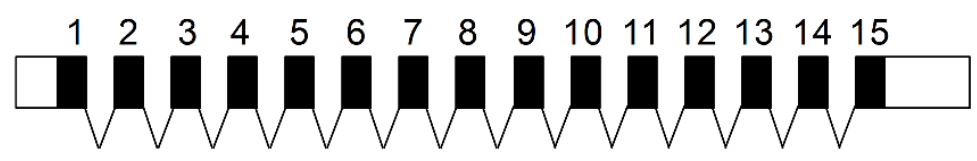

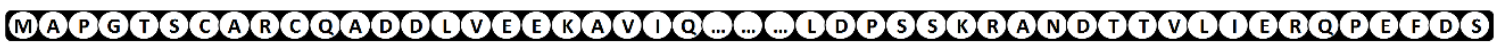

slc11a2- $\beta$

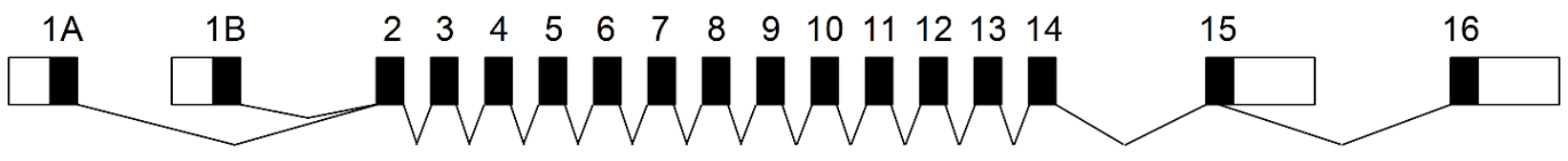

Exon $1 \mathrm{~A}$

Exon 15

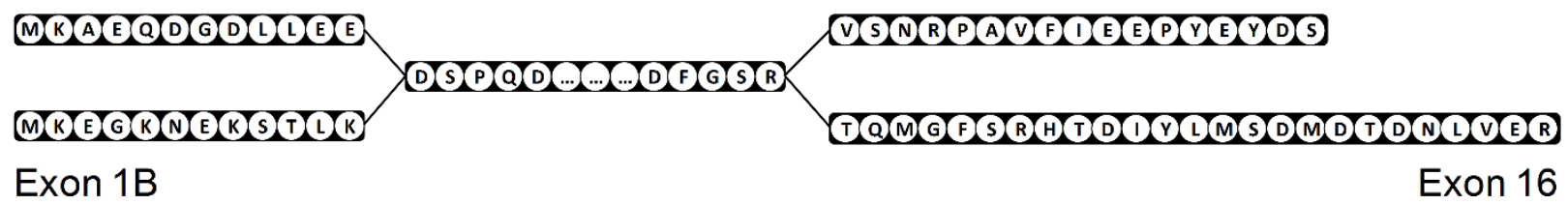

Figure 2 Schematic representation of s/c11a2- $\alpha$ and s/c11a2- $\boldsymbol{\beta}$ transcripts and putative proteins. S/c11a2- $\alpha$ produces a single transcript, encoding a 560 aa protein. S/c11a2- $\beta$ produces 4 transcripts, encoding 4 putative proteins, 2 of 554 aa $(\beta 1$ and $\beta 2$, from exons $1 \mathrm{~A}$ or $1 \mathrm{~B}$ to exon 15 , respectively) and 2 of 562 aa ( $\beta 3$ and $\beta 4$, from exons $1 \mathrm{~A}$ or $1 \mathrm{~B}$ to exon 16 , respectively). Difference in size results from an alternative splice site in exon 15 and replacement of its final 17 aa for 25 aa encoded by exon 16. Exons are represented as black boxes, UTRs as white boxes.

No clear sequences matching the iron responsive element (IRE) motifs, commonly found in mammalian $S L C 11 A 2$, were identified in any of the 3' regions of either slc11a2- $\alpha$ or slc11a2- $\beta$. However, one IRE motif was found in the $5^{\prime}$ region of slc11a2- $\beta$ (5'-UGUCUGUGUCAGAGAACAUGGUG-3'), with an upstream distance to the start codon of 508 bp, using RegRNA $[66,67]$ and corroborated manually.

Full-length genomic sequences of sea bass slc11a2- $\alpha$ and slc11a2- $\beta$ were deposited in GenBank with accession numbers HQ451945 (slc11a2- $\alpha$ ) and HQ451946 (slc11a2- $\beta$ ).

\section{Structure analysis of sea bass Slc11 putative proteins}

To predict the functionality of sea bass Slc11 putative proteins, we compared them with other known SLC11 proteins, and analyzed them using several bioinformatics tools (listed at ExPASy [68]), searching for characteristic features of this protein family.

The complete open reading frame of sea bass slc11a2- $\alpha$ comprises a single transcript of 1683 nucleotides, encoding a putative protein of 560 residues (Figure 2 and Additional File 1, Figure S1), with a predicted molecular mass of 61.6 $\mathrm{kDa}$. The complete open reading frame of sea bass slc11a2- $\beta$ comprises 4 different transcripts, encoding 4 putative proteins (Figure 2 and Additional File 1, Figure S1) that result from the alternative usage of two 5 ' exons encoding distinct $\mathrm{N}$-termini and the alternative splicing of two 3' exons encoding distinct C-termini of the proteins. Two transcripts of 1665 nucleotides, named slc11a2- $\beta 1$ (from exon 1A to exon 15) and slc11a2- $\beta 2$ (from exon $1 \mathrm{~B}$ to exon 15), and two transcripts of 1689 nucleotides, named slc11a2- $\beta 3$ (from exon $1 \mathrm{~A}$ to exon 16) and slc11a2- $\beta 4$ (from exon $1 \mathrm{~B}$ to exon 16), encode putative proteins of 554 and 562 residues, with predicted molecular masses of 61.2 and $62.2 \mathrm{kDa}$, respectively.

The 8 amino acid discrepancy in length results not from the substitution of exon 15 for exon 16, but rather from an alternative splice site in exon 15 . Only the final 17 amino acids encoded by exon 15 (out of 35 ) are replaced by the 25 amino acids encoded by exon 16 (figure 2). Both exon $1 \mathrm{~A}$ and exon $1 \mathrm{~B}$ have the same size and encode the same number of amino acids.

Comparison with other teleost and mammalian species SLC11 proteins showed that the signature features of the SLC11 family $[36,42,45,69]$ can also be found in the sea bass (Figure 3 and Additional File 1, Figure S1): twelve putative transmembrane domains (TMD), a conserved transport motif (between TMD8 and TMD9), cysteine residues in loops 2, 5 and 7 . Other motifs 


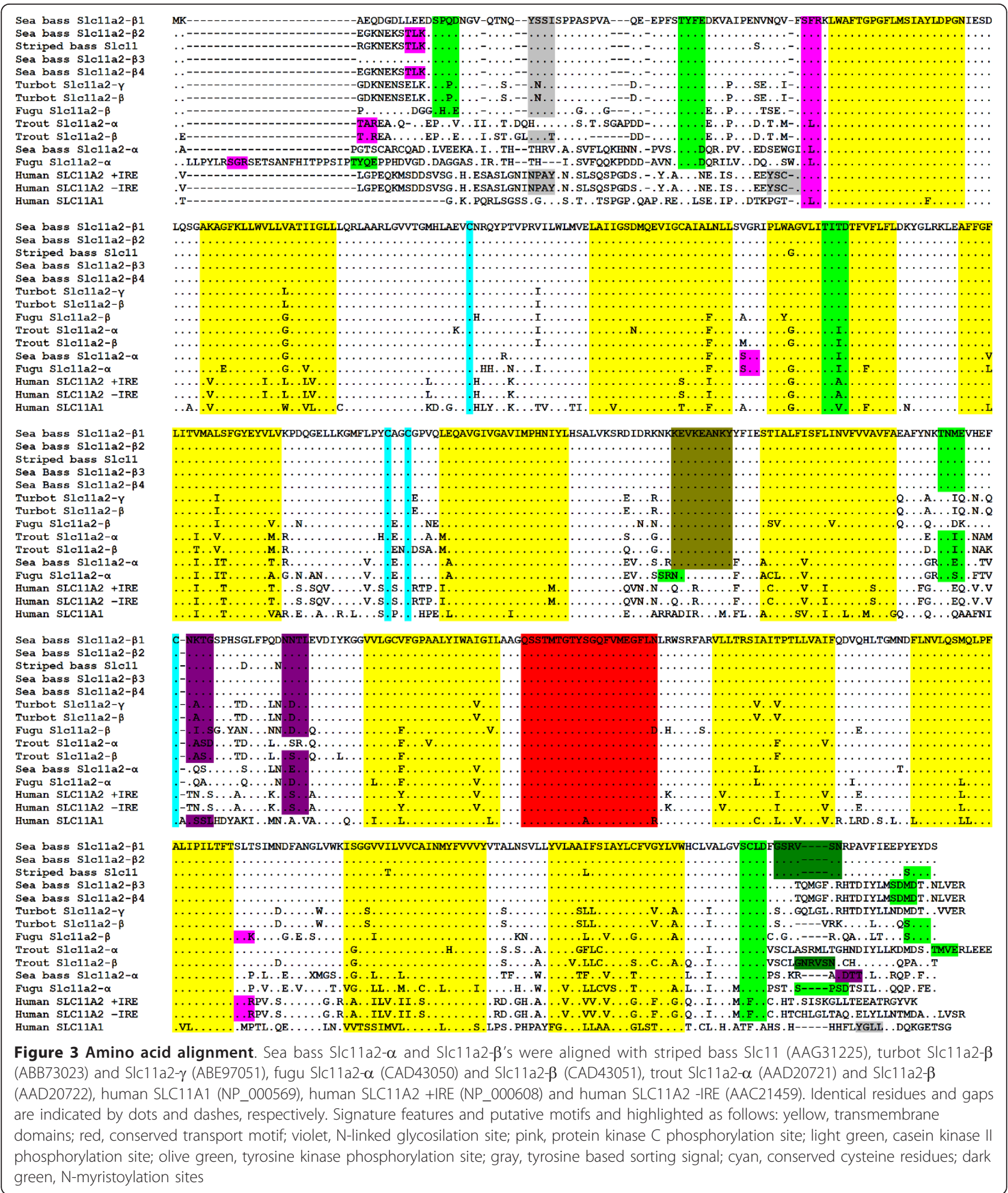

observed in previously described SLC11 proteins, including some exclusive to teleost fish, are also present in sea bass. Two N-linked glycosylation sites (N-X-S/T-X), located in loop 7 , are present in all Slc11a2- $\beta$ forms, but only one is present in Slc11a2- $\alpha$ loop 7, with another

located in the $\mathrm{C}$ terminus. A conserved protein kinase $\mathrm{C}$ phosphorylation site $(\mathrm{S} / \mathrm{T}-\mathrm{X}-\mathrm{R} / \mathrm{K})$ was also found immediately before TMD1, and one other was only found to be shared by sea bass Slc11a2- $\alpha$ and fugu Slc11a- $\alpha$, in loop 3. A third protein kinase $C$ phosphorylation site 
was found to be present in the 5 ' cytoplasmatic extremity of sea bass Slc11a2- $\beta 2$ and Slc11a2- $\beta 4$, striped bass Slc11, fugu Slc11a2- $\alpha$ and trout Slc11a2- $\alpha$ and $\beta$. Six, five and four casein kinase II phosphorylation sites (S/ T-XX-D/E) were found in Slc11a2- $\beta(3,4)$, Slc11a2- $\beta(1,2)$ and Slc11a2- $\alpha$, respectively. One tyrosine kinase phosphorylation site (R/K-XX( or XXX)-D/E-XX(or XXX)-Y) in loop 6 was found to be conserved in teleosts, with the exception of fugu Slc11a- $\alpha$. One tyrosine based sorting signal (NPXY or YXXФ; where $\Phi$ is a bulky hydrophobic residue) in the $\mathrm{N}$ terminus was also found to be conserved in teleosts, with the exception of the alpha forms of trout, sea bass and fugu. Several N-myristoylation sites (G-\{EDRKHPFYW\}-XX-[STAGCN]-\{P\}) were also found to be conserved among all Slc11a2- $\beta$ forms (data not shown), but one site was only found to be present in the 3' cytoplasmatic extremity of sea bass Slc11a1- $\beta(1,2)$, as well as striped bass Slc11 and Rainbow trout Slc11a2- $\beta$ (figure 3). All phylogenetic analysis, performed with the maximum-likelihood, maximumparsimony, neighbor-joining and Bayesian inference methods were found to be consensual, placing sea bass Slc11a2- $\beta$ clustered with other fishes Slc11a2- $\beta$ and Slc11 (Figure 4 and Additional File 3, Figure S3) and sea bass Slc11a2- $\alpha$ clustered with other fishes Slc11a2- $\alpha$, with the exception of trout Slc11a2- $\alpha$. As with all other teleost fish Slc11 homologs described so far, sea bass Slc11 homologs are also closer to mammalian SLC11A2 than to SLC11A1.

\section{Paralogy and synteny}

To determine the evolutionary history of the SLC11 gene family in vertebrates, we explored in detail the genomic "context" of both human SLC11A1 and SLC11A2 paralogs. Other authors [36] have previously suggested that SLC11A1 and SLC11A2 genes might have originated from the duplications of the genome in vertebrate ancestry, the so-called $2 \mathrm{R}$, given their location to the Hox chromosomes in Hsa2 and Hsa12 (Figure $5 \mathrm{~A}$ ). We find strong support for this hypothesis. Within a $1 \mathrm{Mb}$ window in the vicinity of the SLC11A1 and SLC11A2 genes, various gene families have paralog members mapping to expected Hox chromosome regions. That is the case of TMBIM1, which maps close to the SLC11A1 paralog in Hsa2, while FAIM2 maps to Hsa12. TNS1 maps to the left end of the window of the SLC11A1 gene, and has paralogs mapping to the three other human Hox genome locations. In the case of the SLC11A2 gene, 6 gene families show a consistent duplication pattern, with paralogs mapping to expected regions of Hox-linked paralogy, namely $\mathrm{Hsa} 2 \mathrm{q}$ (Figure $5 \mathrm{~A}$ ).

In the analysed teleosts a similar SLC11 gene complement is also found (with the exception of $D$. rerio).
However, the precise orthology/paralogy relationships to the mammalian counterparts are yet to be firmly established. While the phylogeny clearly indicates that both fish genes strongly group with tetrapod SLC11A2 (Figure 4 Additional File 3, Figure S3), other features (e.g. subcellular localization) make the orthology assignment more contentious [36]. Syntenic data can be a powerful tool to facilitate the finding of orthologs and in the identification of duplication processes [70]. The abundance of genome data from teleosts allows us the use of mapping data to clarify the phylogenetic findings. We have analysed the genome locations and gene environment of the teleost SLC11 genes in zebrafish (Danio rerio), fugu (Takifugu rubripes), tetraodon (Tetraodon nigroviridis), medaka (Oryzias latipes) and stickleback (Gasterosteus aculeatus) (Figure $5 \mathrm{~B}$ ). We find a strong degree of conservation between the gene arrangements of various fish species. The vicinity of the teleost SLC11 loci have their human ortholog equivalents mapping to the SLC11A2 chromosome, Hsa12q. Thus, we conclude that in agreement with the phylogenetic data, the genomic location of the teleost genes indicates that they are both SLC11A2-like. Furthermore, we observe that the mechanistic origin of the SLC11 genes is likely linked to the fish $3 \mathrm{R}$ genome duplication [71]. We find in the vicinity of both fish isoforms, gene families with duplicates mapping to both SLC11 fish chromosomal regions. For example, we noticed that AGAP2 gene family in medaka has one member mapping to chromosome 7 (along with one of the SLC11 isoforms), while a second isoform maps in chromosome 5 with the second isoform of teleost SLC11. The phylogenies of the genes families with duplicated members show that the duplication timing dates back to the origin of the teleosts (Additional File 4, Figure S4). Thus, we can safely conclude that the teleost genes are of the SLC11A2 type (which we name slc11a2- $\alpha$ and slc11a2- $\beta$ ), and resulted from the fish specific genome duplication.

\section{Constitutive expression of s/c11a2- $\alpha$ and s/c11a2- $\beta$ in sea bass tissues}

To gain some insight into sea bass slc11 basic functions, mRNAs constitutive expression of both forms was determined in relevant tissues. Constitutive expressions of slc11a2- $\alpha$ and slc11a2- $\beta$ (all 4 isoforms) were evaluated by real-time PCR in several tissues, namely liver, spleen, head/trunk kidney, gill, brain, stomach, pyloric ceca, anterior/mid/posterior sections of the intestine and rectum (Figure 6). The liver was found to be the organ with the highest overall expression of the slc11a2 genes, followed by the mid and posterior portions of the intestine, stomach and spleen. Regarding the contribution of each slc11a2 form in the different tissues, there is a clear predominance of 


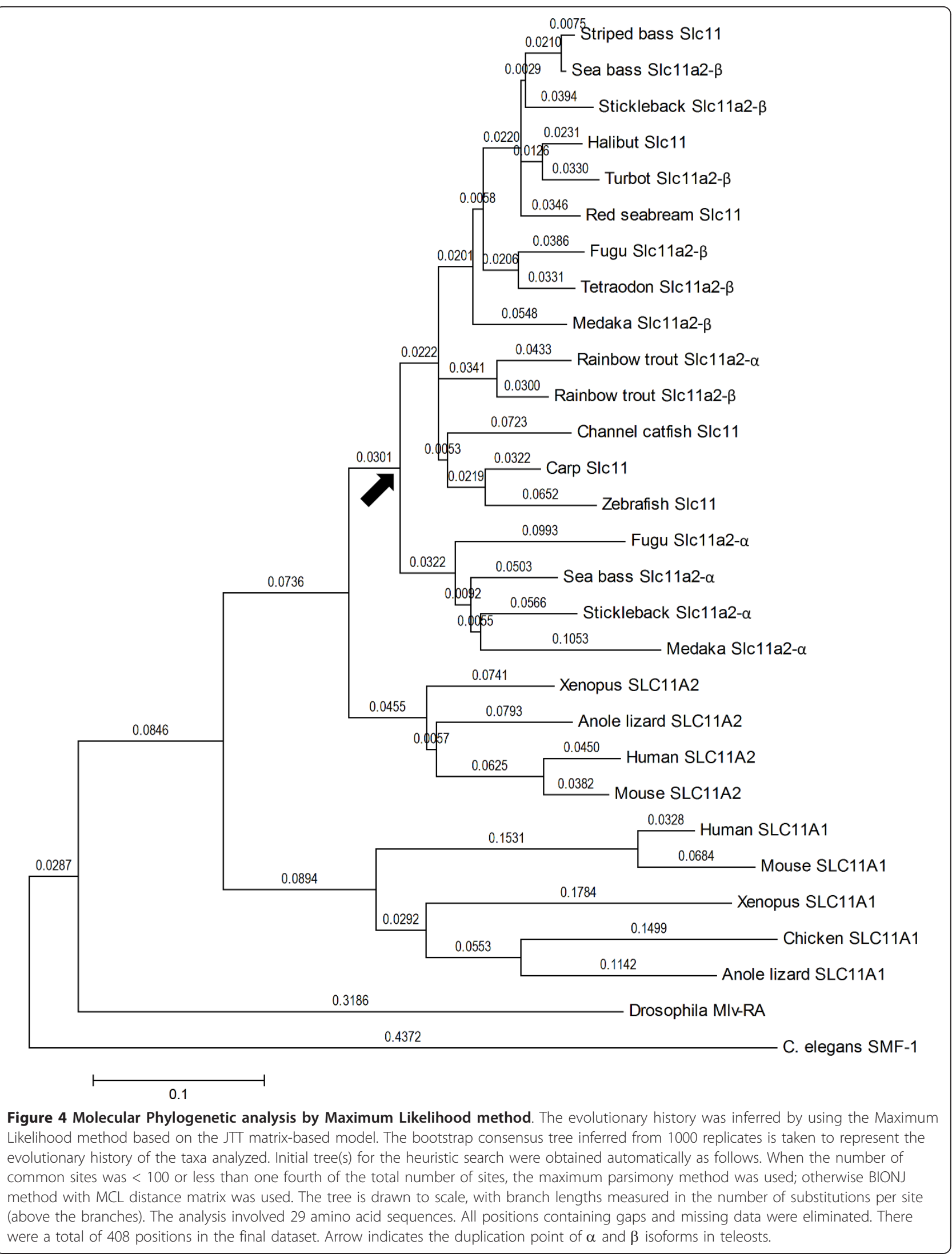




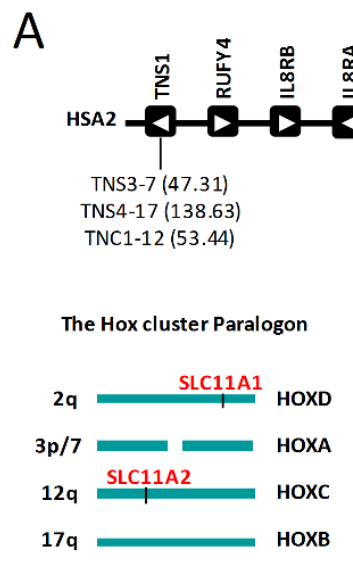

B

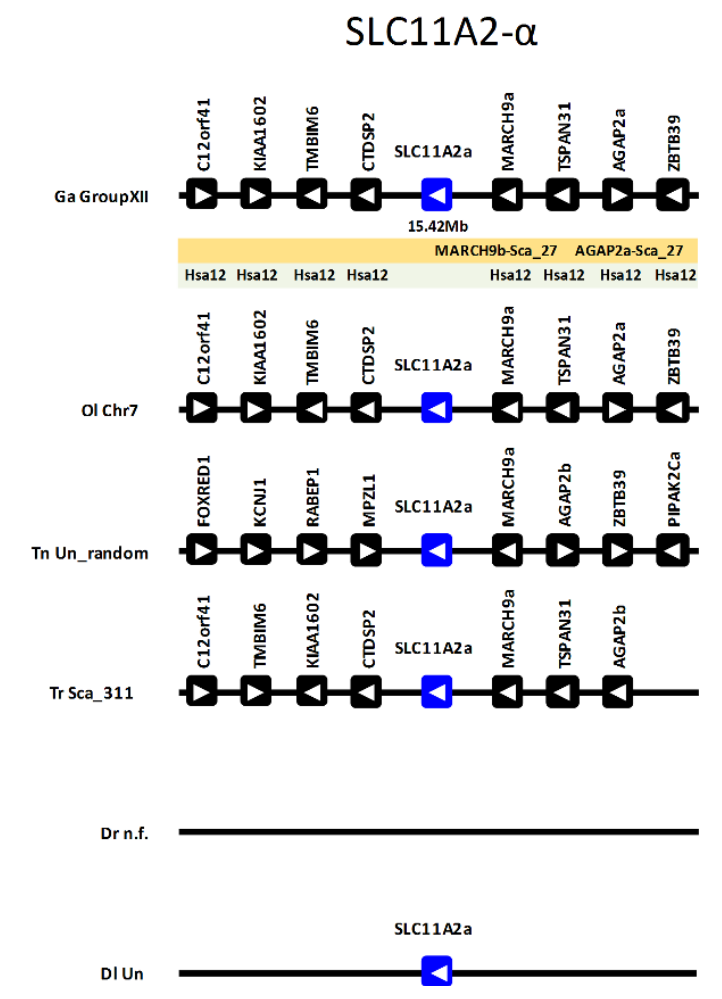

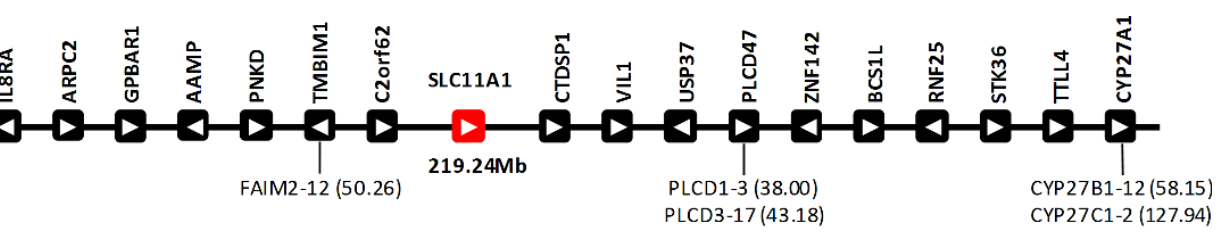
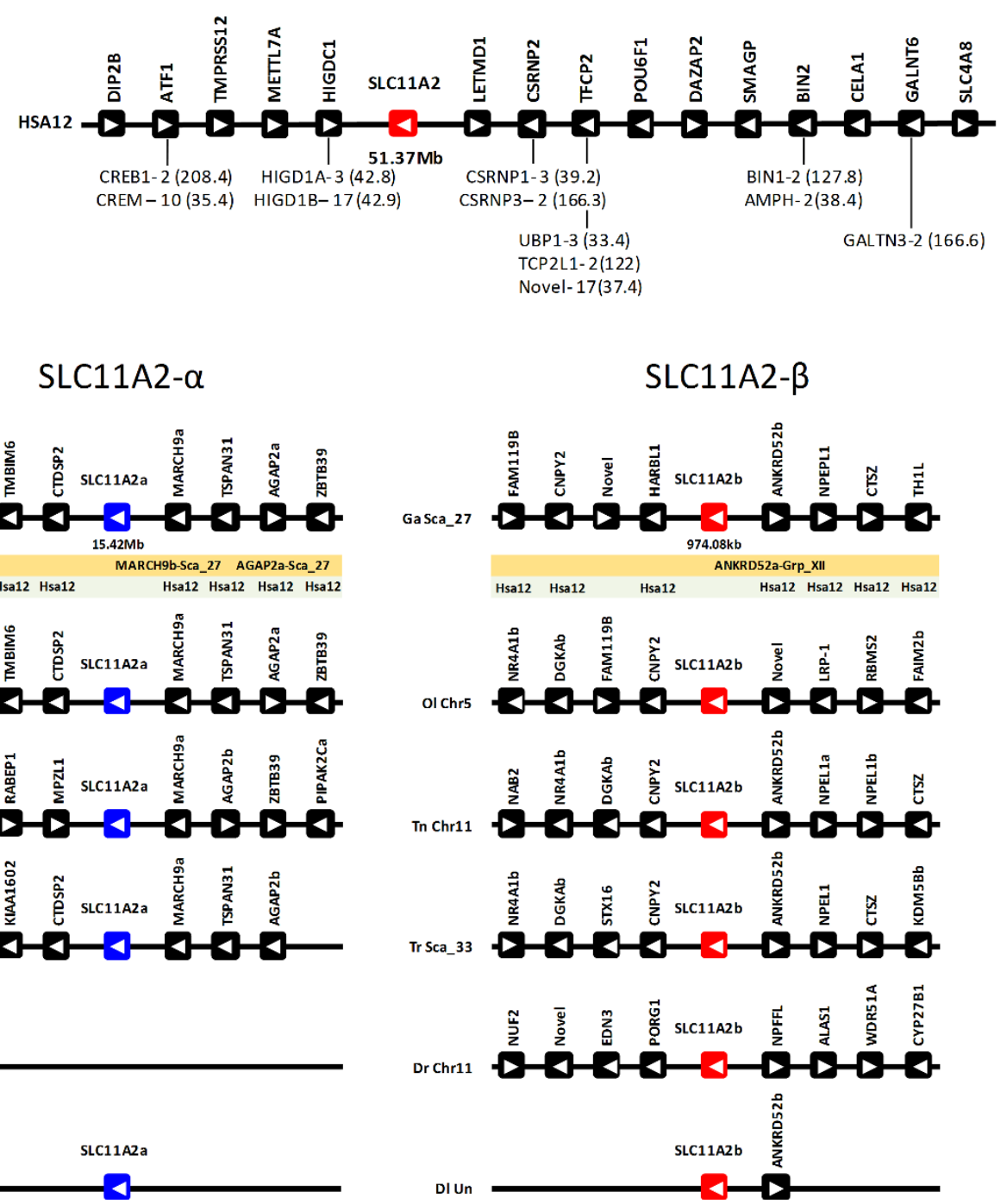

Figure 5 Chromosomal location of the human SLC11A1 and SLC11A2 genes (A) and SLC11 gene loci in teleost species (B). (A) Paralogs of gene families with multiple members are shown below each ORF, with distance in $\mathrm{Mb}$ to the $\mathrm{p}$ telomere of the respective chromosome. (B) Below the G. aculeatus loci, the location of teleost specific 3R paralogs is shown; also the genomic mapping position of human orthologs is presented. Ga - Gasterosteus aculeatus, Dr - Danio rerio, Ol - Oryzias latipes, Tn - Tetraodon nigroviridis, Tr - Takifugu rubribes, DI - Dicentrarchus labrax. Arrows denote gene orientation.

slc11a2- $\beta$ in the liver, spleen, head kidney, rectum, gill and brain and a predominance of slc11a2- $\alpha$ in the stomach, pyloric ceca and all portions of the intestine.

Constitutive expression of the four isoforms of s/c11a2- $\beta$ by semi-quantitative RT-PCR

In order to assess the relative contribution of each $\beta$ isoform to the overall slc11a2- $\beta$ constitutive expression, a more thorough analysis was performed. Relative constitutive expressions of the four isoforms of sea bass slc11a2- $\beta$ were determined by semi-quantitative PCR, since the distance between alternative 5' and 3' exons is too great to adequately use real-time PCR (Figure 7). The four isoforms were named $\beta 1$ (from exon $1 \mathrm{~A}$ to exon 15), $\beta 2$ (from exon $1 \mathrm{~B}$ to exon 15), $\beta 3$ (from exon $1 \mathrm{~A}$ to exon 16) and $\beta 4$ (from exon $1 \mathrm{~B}$ to exon 16 ). 


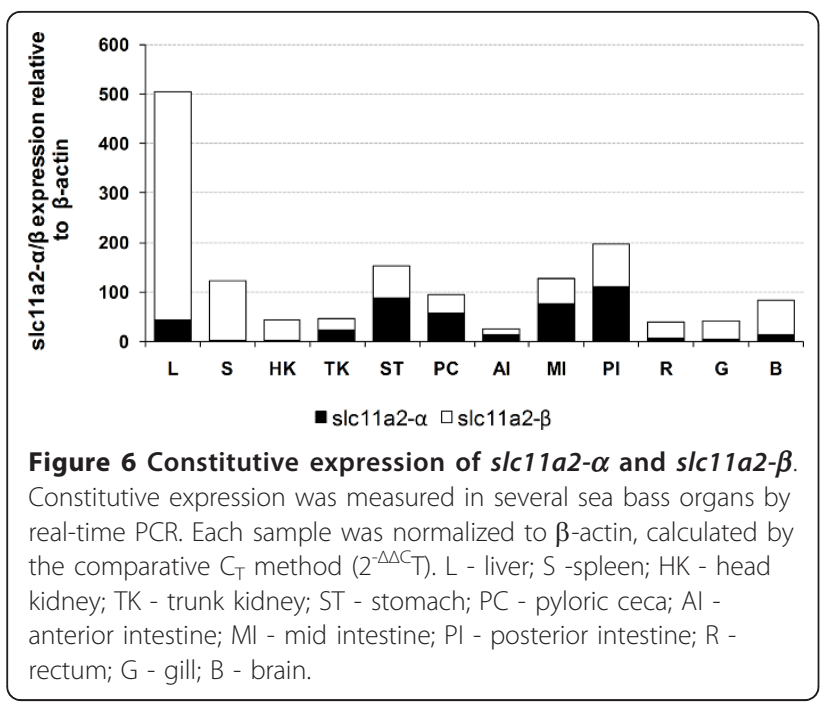

In most of the tested tissues, there is a predominance of the slc11a2- $\beta 1$ form, followed by slc11a2- $\beta 2$. Slc11a2$\beta 3$ has a lower constitutive expression in most tissues, but presents significant expression in the head kidney, stomach and rectum, whereas slc11a2- $\beta 4$ is the form with the lowest expression, although it represents $26 \%$ of the overall expression of slc11a2- $\beta$ in the gill.

\section{In situ hybridization}

An in situ hybridization was performed in several tissues to identify the subcellular distribution of sea bass $s l c 11$ mRNAs, to obtain further information useful in establishing parallelisms with their mammalian homologous counterparts, and to reiterate the results observed in the constitutive expression analysis.

In the liver (Figure $8 \mathrm{~A}-\mathrm{D}$ ), slc11a2- $\alpha$ mRNA is very scarce, being detected in few hepatocytes whereas slc11a2- $\beta$ in abundantly slc11a2- $\alpha$ mRNA is scarce, being only conspicuous in the melanomacrophage centers. Slc11a2- $\beta$ on the other hand is abundant not only in the melanomacrophage centers but also in the spleen's white pulp. In the head kidney (Figure $8 \mathrm{I}-\mathrm{L}$ ), both slc11a2- $\alpha$ and slc11a2- $\beta$ have a similar distribution, being abundant in the melanomacrophage centers and the surrounding lymphomyeloid tissue, and to a much lesser degree in the hematopoietic tissue. In the intestine, slc11a2- $\alpha$ and slc11a2- $\beta$ mRNA present similar patterns of distribution in the anterior (Figure $8 \mathrm{M}-\mathrm{P}$ ) and mid section (Figure $8 \mathrm{Q}-\mathrm{T}$ ). Slc11a2- $\alpha$ is found to be mostly concentrated in the apical (brush border) membrane of the enterocytes, whereas slc11a2- $\beta$ presents a more homogeneous distribution, not only in enterocytes but also in other intestinal cells. However, in the posterior intestine (Figure $8 \mathrm{U}-\mathrm{X}$ ), both forms of slc11a2 seem to be accumulated in or around the goblet cells, with limited presence in the enterocytes.

In all tissues, hybridization with sense probes (control) for either slc11a2- $\alpha$ or slc11a2- $\beta$ produced no significant staining.

Hematological parameters and tissue iron content in the in vivo experimental models

Several hematological and iron parameters were measured to validate the models of in vivo experimental

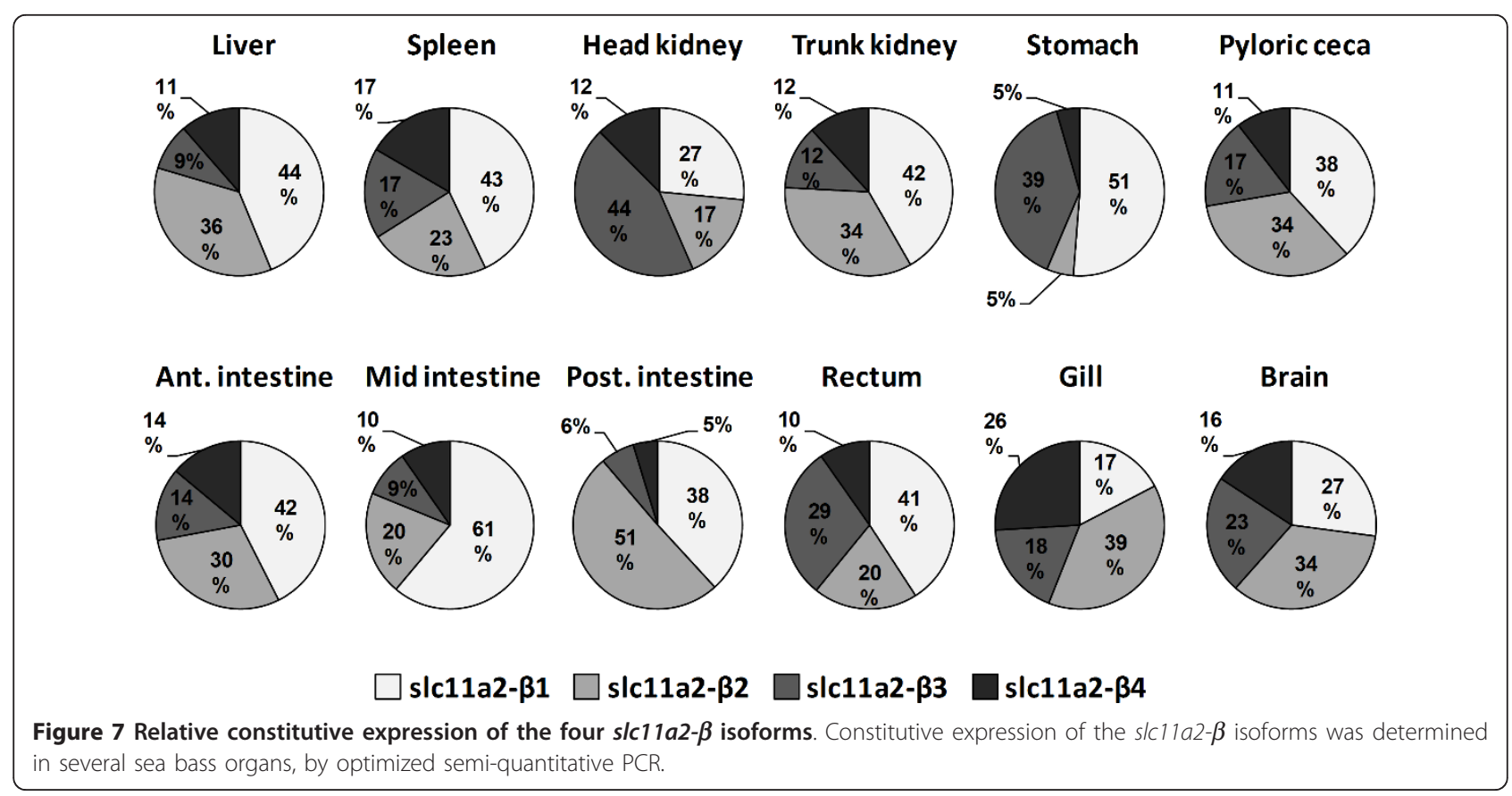




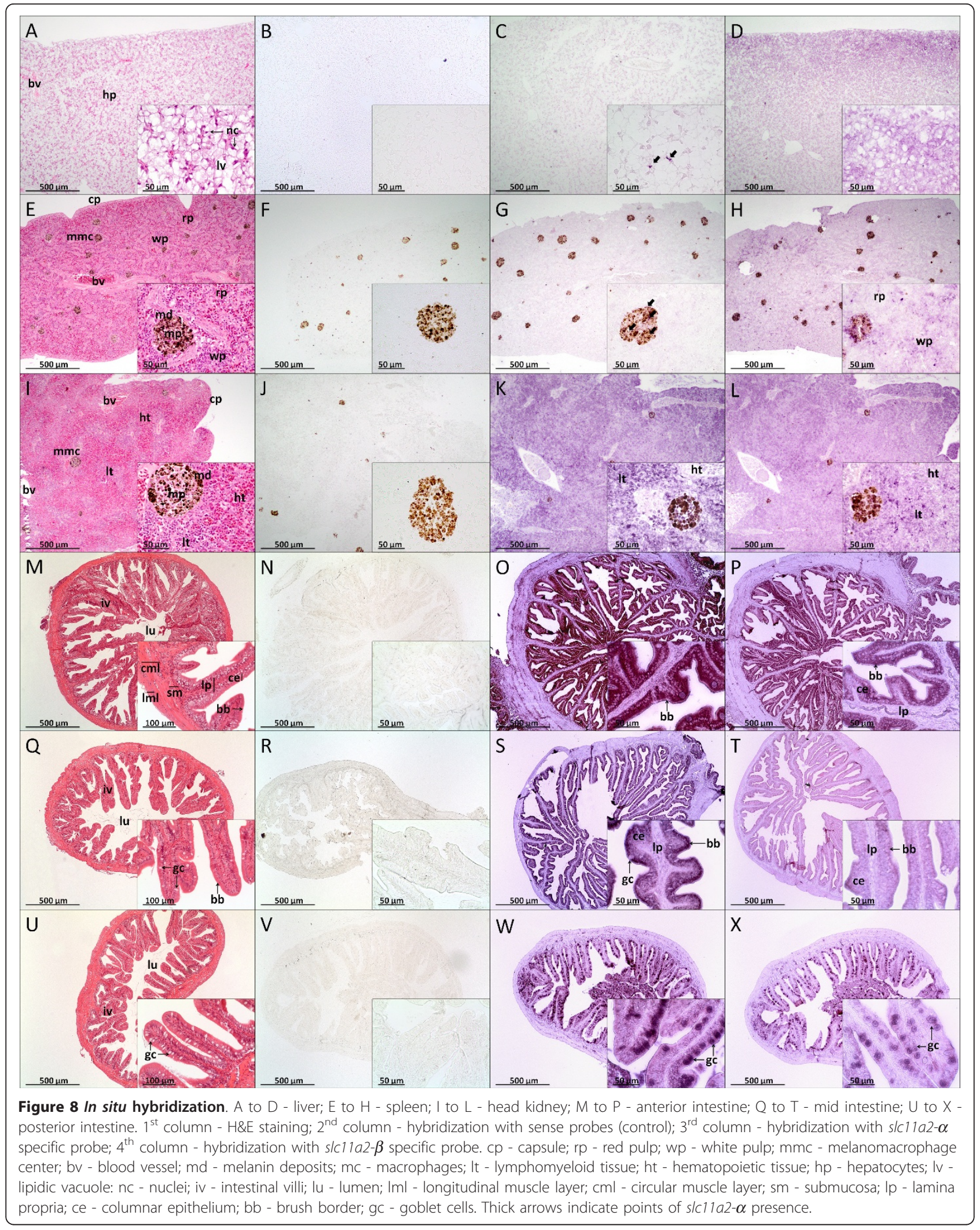


iron modulation or infection with Photobacterium damselae spp. piscicida. During the experimental infection, mortality was monitored and found to be high, but with fish surviving past the final experimental time point, indicating a chronic infection (Additional File 5, Figure S5).

In the iron overloaded animals, no changes were observed in hematocrit (Figure 9 A), and a small but significant increase was observed in the RBC count during the course of the experiment (Figure $9 \mathrm{~B}$ ). Increases in serum iron and transferrin saturation (Figure $9 \mathrm{C}-\mathrm{D}$ ) were also observed, the highest levels at 4 days after overload, with decreases during the course of the experiment and recovery to control levels at 14 days. Liver iron levels were increased (Figure $9 \mathrm{E}$ ), with the maximum value at 7 days post-modulation and a decrease towards 14 days, but still over 3 times higher than the control animals. In the iron deficient animals, significant decreases of all hematological parameters were observed during the course of the experiment, with values remaining below the control levels (Figure 9 A-D). Liver iron levels did not differ significantly from control animals during the experimental iron deficiency (Figure $9 \mathrm{E}$ ).

Significant and steady decreases of all hematological parameters were observed during the course of the infection (Figure 9 F-I). Liver iron levels increased slightly but not significantly from control animals (Figure $9 \mathrm{~J}$ ).

\section{Slc11a2 expression in the in vivo models of iron modulation and infection}

In order to determine the potential involvement of sea bass slc11a2- $\alpha$ and slc11a2- $\beta$ in the iron metabolism and immune response, we evaluated their expression under conditions of iron modulation (overload or deficiency) or bacterial infection.

In the experimental iron modulation model, slc11a2- $\alpha$ and slc11a2- $\beta$ expressions were initially evaluated in the liver, spleen, head kidney and intestine, 4 days after iron modulation (Figure $10 \mathrm{~A}-\mathrm{B}$ ). In iron deficiency, there was a significant increase of slc11a2- $\alpha$ expression in the intestine, with no major changes in the other tested organs. No significant changes were observed in slc11a2- $\beta$ expression. In iron overload, no significant changes in slc11a2- $\alpha$ were observed, whereas slc11a2- $\beta$ expression was found to be increased in the liver. Slc11a2- $\alpha$ and slc11a2- $\beta$ expressions were subsequently evaluated in the liver at 4, 7 and 14 days after iron modulation (Figure 10 D-E). As before, no significant changes were observed in slc11a2- $\alpha$ expression to either condition in the liver, whereas slc11a2- $\beta$ was found $t$ be significantly up-regulated at 4 days post iron overload (over 14-fold), decreasing during the course of the experiment to near control values, but still over 2-fold higher. Expression of slc11a2$\alpha$ and slc11a2- $\beta$ was also evaluated 4 days after iron modulation in several sections of the digestive tract (Figure $10 \mathrm{C}$ ), in order to identify the portion of the intestine responsible for the response of slc11a2- $\alpha$. Significant increases of slc11a2- $\alpha$ expression were observed in the mid and posterior sections of the intestine, with no significant changes in the anterior section or rectum.

In the experimental model of infection, slc11a2- $\alpha$ and slc11a2- $\beta$ expressions were measured in the liver, spleen and head kidney by real-time PCR, 24, 48, 72 and $96 \mathrm{~h}$ after infection. In the liver (Figure $11 \mathrm{~A}$ ), a steady increase in slc11a2- $\beta$ expression was observed during the course of the infection, with the maximum value at $72 \mathrm{~h}$ post-infection (approximately 6.8-fold increase), followed by a recovery to control levels at 96 $\mathrm{h}$. In the spleen (Figure $11 \mathrm{~B}$ ), an increase of slc11a2- $\beta$ was also observed during the course of the infection, accompanied by a recovery to control levels at $96 \mathrm{~h}$. In the head kidney (Figure $11 \mathrm{C}$ ), a significant increase of slc11a2- $\beta$ was observed $24 \mathrm{~h}$ after infection and was maintained during its course. No significant changes were observed in slc11a2- $\alpha$ expression in any of the tested organs.

\section{SIc11a2 expression in the in vitro models of iron overload and infection}

We investigated the role of slc11a2- $\alpha$ and slc11a2- $\beta$ in the iron metabolism and immune response at a cellular level, by using leukocytes from spleen, one of the most important erythropoietic and immune organs in fish.

In the iron overload in vitro model, expression of slc11a2- $\alpha$ and slc11a2- $\beta$ in leukocytes was measured by real-time PCR at $0,6,12,24,48$ and 72 hours after the addition of ferric ammonium citrate (Figure $12 \mathrm{~A}$ ). No significant changes were observed in slc11a2- $\alpha$ during the course of the experimental iron overload. Slc11a2- $\beta$, on the other hand, increased significantly from $6 \mathrm{~h}$ postinfection (about 2.5-fold), reaching the maximum increase at $24 \mathrm{~h}$ (about 8.5 -fold) and decreased at $48 \mathrm{~h}$, returning to control levels at $72 \mathrm{~h}$.

In the in vitro infection model with heat-inactivated P. damselae, expression of slc11a2- $\alpha$ and slc11a2- $\beta$ in leukocytes was also measured by real-time PCR at 0,6 , $12,24,48$ and 72 hours after infection (Figure 12 B). No significant changes were observed in slc11a2- $\alpha$ during the course of infection. Slc11a2- $\beta$, on the other hand, increased significantly at $6 \mathrm{~h}$ post-infection (about 8fold), followed by a decrease at $12 \mathrm{~h}$ (to about 4-fold) and returned to control levels at $24 \mathrm{~h}$.

\section{Discussion}

In the present study, we set out to analyse the evolutionary and functional patterns of $S L C 11$ genes in vertebrates, in particular teleosts. We have used the European sea bass (Dicentrarchus labrax) as a model and have 


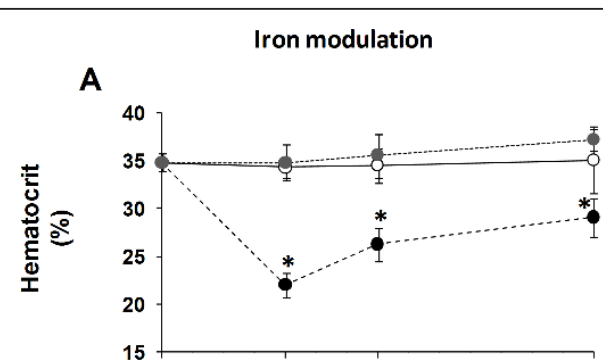

B

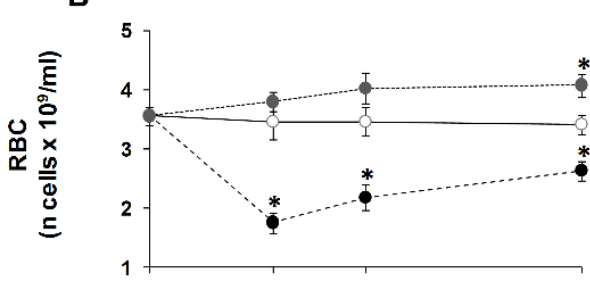

C

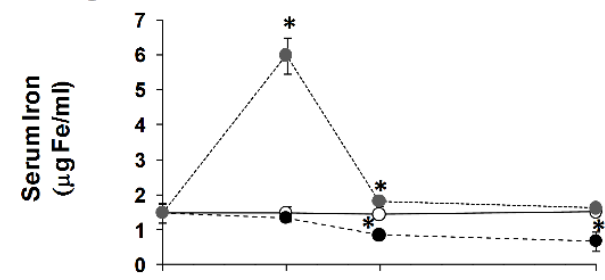

D

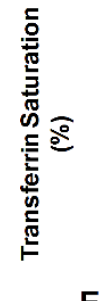

E

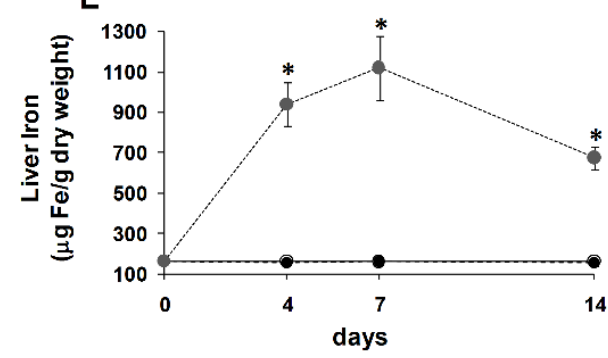

-O- Control

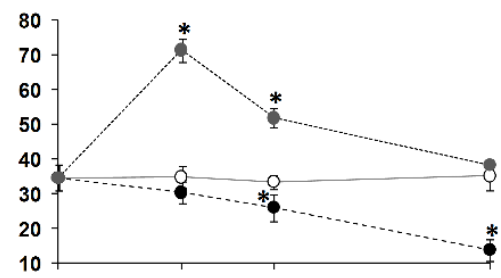

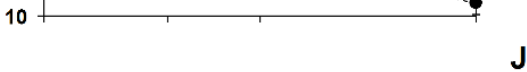

F

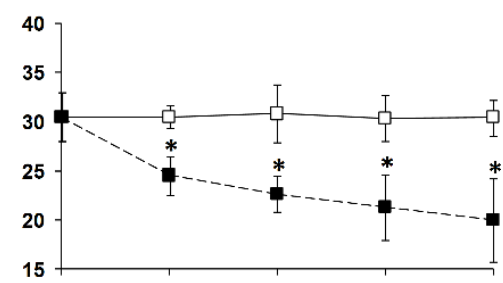

G

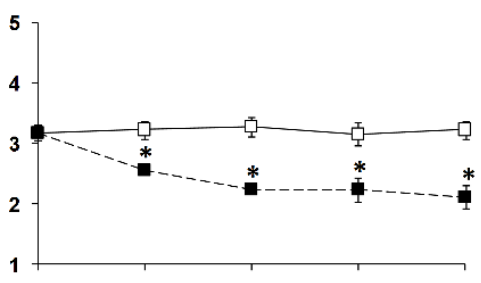

$\mathrm{H}$

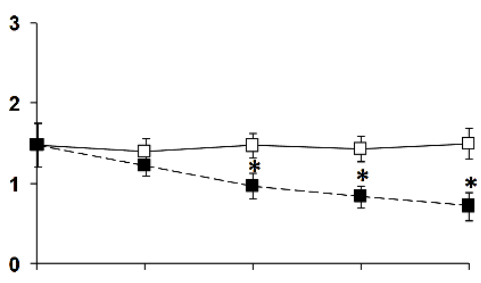

I
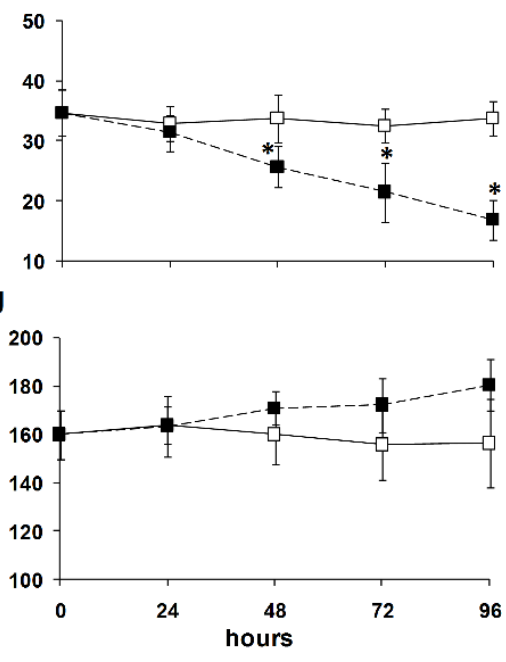

Control

Infected

Figure 9 Hematological parameters kinetics in iron modulated and infected fish. Iron modulation: (A) hematocrit, (B) red blood cells count (RBC), (C) serum iron, (D) transferrin saturation and (E) liver iron; Infection: (F) hematocrit, (G) red blood cells count (RBC), (H) serum iron, (I) transferrin saturation and (J) liver iron. Values are expressed as means \pm S.D. ( $n=5$ or $n=6$ for iron modulation or infection, respectively).

Samples were collected at 4,7 and 14 days after iron modulation or 24, 48, 72 and 96 hours post-infection. Untreated fish were used as a 0-day control $(n=5)$. Differences from the controls were considered significant for ${ }^{*} p<0.05$.

successfully isolated two slc11 genes, named slc11a2- $\alpha$ and slc11a2- $\beta$.

A single transcript was found to be produced, for the slc11a2- $\alpha$ gene, whereas slc11a2- $\beta$ can produce up to four transcripts. The four $\beta$ transcripts are the result of alternative exon splicing in the 5' and 3' ends, in a similar fashion to what has been described for human SLC11A2 [62,63]. But unlike human SLC11A2, where the differences in the 3' end result from alternate exon usage [63], in sea bass the differences result from an 


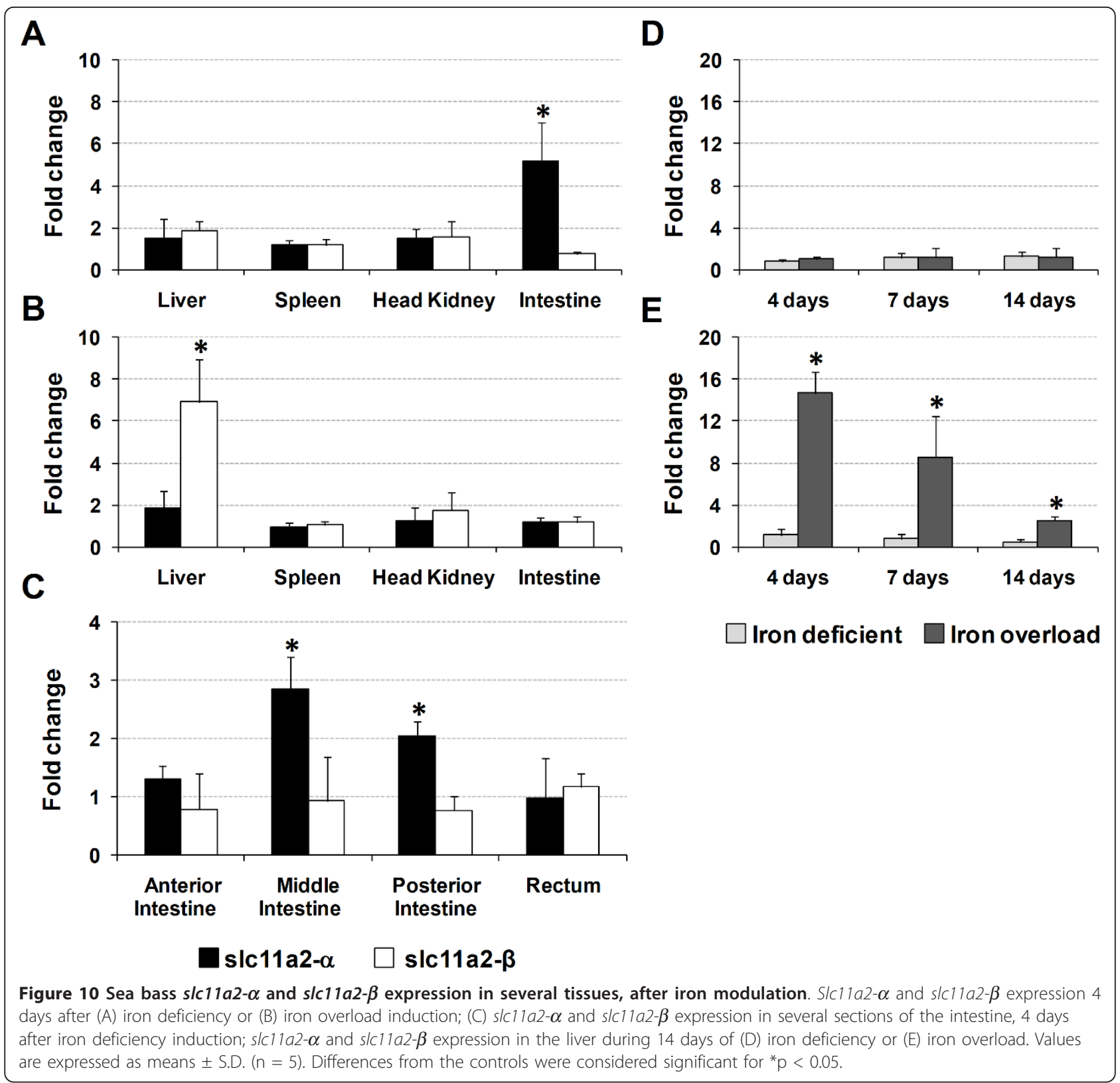

alternative splice site in exon 15 . Thus, only the second half of exon 15 is replaced by exon 16 .

Neither sea bass slc11a2- $\alpha$ nor slc11a2- $\beta$ were found to present a potential iron responsive regulatory protein binding site (IRE) in the 3' UTR, a feature that in contrast is present in mammalian $S L C 11 A 2$ genes $[23,63]$ and has also been described in some teleost fish, such as fugu [36] and common carp [42]. It is, however, absent in other teleosts, such as turbot [35], channel catfish [43] and Japanese flounder [33]. In the SLC11 gene family, IREs have only been described in the 3'-UTR but unexpectedly, an IRE motif was found in the 5 ' region of slc11a2- $\beta$, albeit not in the 5'-UTR itself but rather
205 bp upstream the start of the 5'-UTR. The significance of this finding is still unknown, although as with other 5' IREs, it may be involved in translation repression $[72,73]$.

The single sea bass slc11a2- $\alpha$ transcript encodes for a single functional putative protein, whereas the four slc11a2- $\beta$ transcripts encode for four putative proteins. The four $\beta$ proteins differ in the $\mathrm{N}$ and $\mathrm{C}$ termini, as a result of the encoding by the two alternative exons 1 and the alternative splice site in exon 15 . Since both N and $C$ termini are cytoplasmic, they may be involved in post-transcriptional regulatory processes and in the subcellular localization of these proteins, as occurs in 

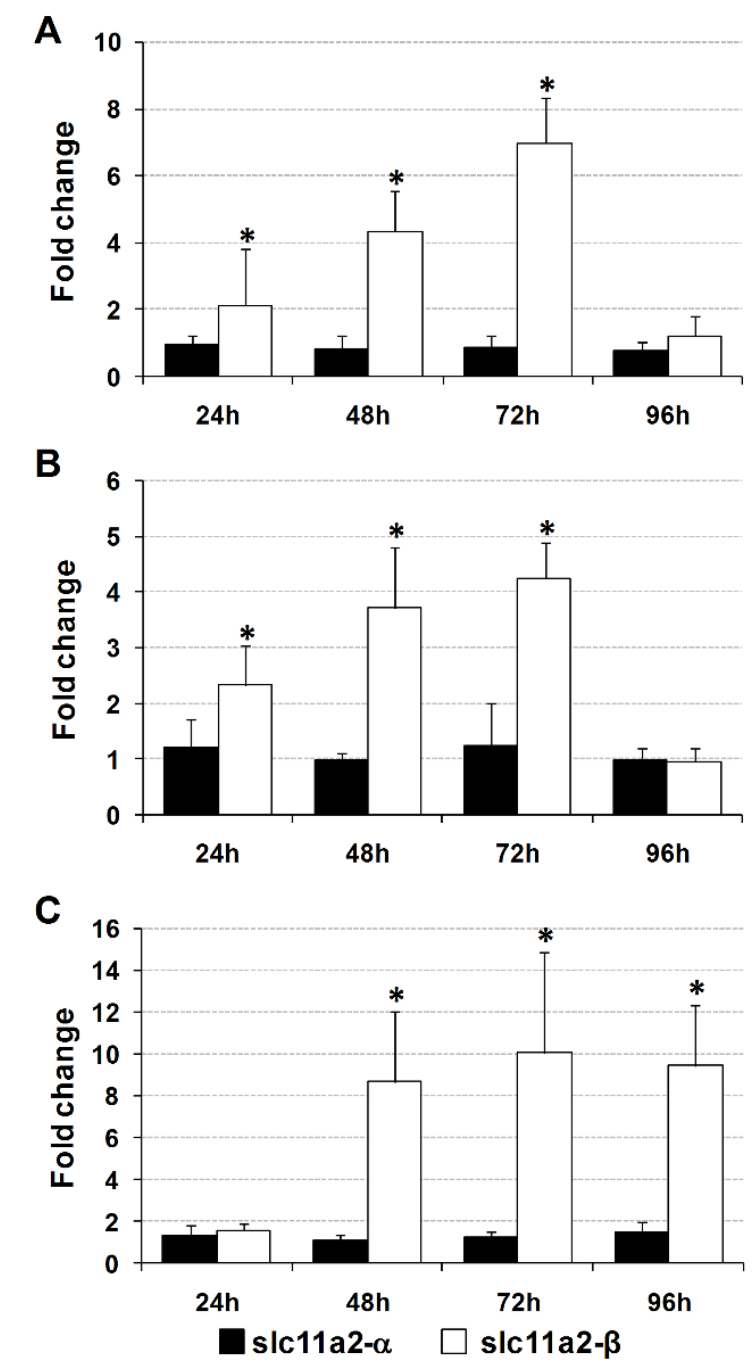

Figure 11 Sea bass slc11a2- $\alpha$ and slc11a2- $\beta$ expression under experimental infection, in the (A) liver, $(B)$ spleen and (C) head kidney. Values are expressed as mean fold change \pm S.D. $(n=5)$. Samples were collected at 24, 48, 72 and 96 hours post-infection with Photobacterium damselae $\left(1.0 \times 10^{5}\right.$ CFU/fish). $\beta$-actin was used as the housekeeping gene. Differences from the control group were considered significant at ${ }^{*} p<0.05$

mammalian SLC11A2 [74]. All sea bass Slc11a2 proteins present the characteristic features of the SLC11 gene family: 12 transmembrane (TM) domains, a consensus transport motif (CTM), several glycosylation and phosphorylation sites and conserved cysteine residues. Multiple alignments clearly show that these features are highly conserved and are very similar to those observed not only in other teleost fish, but also in mammals. However, there are some disparities in the features found in the 5' and 3' endings, namely some N-myristoylation and phosphorylation sites that are not present in all sea bass Slc11a2- $\beta$ forms, and which may also

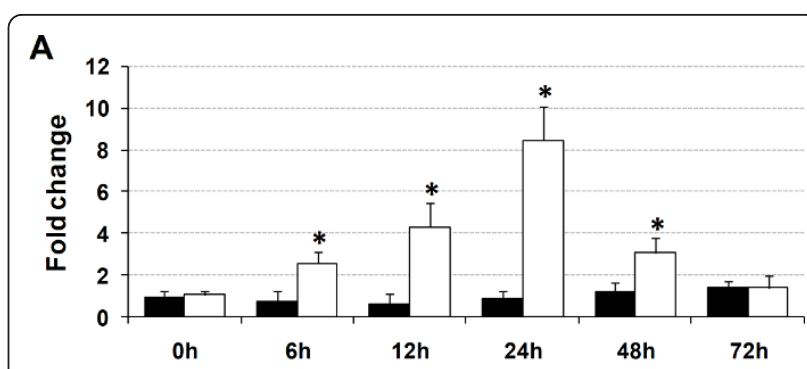

\section{B}

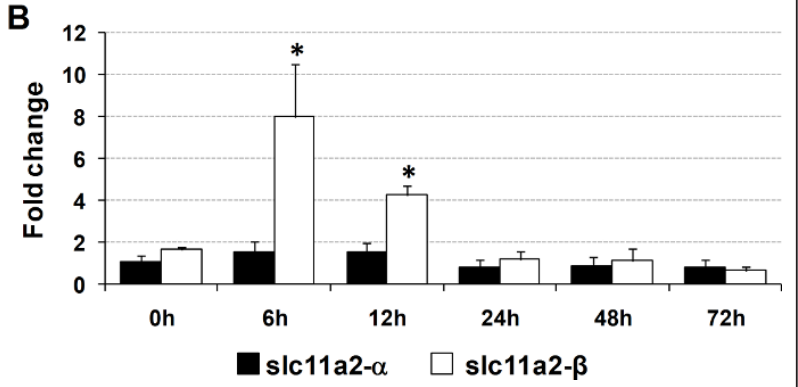

Figure 12 Expression of s/c11a2- $\alpha$ and s/c11a2- $\beta$ in sea bass leukocytes under experimental (a) iron overload or (b) infection, in vitro. Samples were collected at 0, 6, 12, 24, 48 and 72 hours after addition of ferric ammonium citrate (iron overload) or heat-inactivated $P$. damselae (infection). Values are expressed as mean fold change \pm S.D. $(n=5)$. $\beta$-actin was used as the housekeeping gene. Differences from the control group were considered significant at ${ }^{*} p<0.05$.

contribute to a differential post-transcriptional regulation and subcellular localization. In all the tree models, produced by maximum-likelihood, neighbour-joining, maximum parsimony or Bayesian inference, phylogenetic analysis places all sea bass Slc11 proteins clustered with other teleost fish Slc11 proteins, which in turn cluster with mammalian SLC11A2 proteins, clearly separated from SLC11A1. Among teleost proteins, there are two clear separate clusters. One cluster encompasses sea bass Slc11a2- $\beta$ protein as well as all other Slc11 and Slc11a2- $\beta$ proteins. The other cluster includes sea bass Slc11a2- $\alpha$ and all other $\alpha$ proteins, with the exception of rainbow trout Slc11a2- $\alpha$, suggesting that rainbow trout Slc11a2- $\alpha$ may have a different evolutionary origin than other Slc11a2- $\alpha$ proteins.

There are some explanations that could account for the existence of two closely related slc11a2 genes in sea bass, which are in agreement with what has already been proposed for other teleost fish, such as fugu. The current evolutionary scenario proposes that slc11 paralogs appeared through the $2 \mathrm{R}$ genome duplication $[36,49,50]$, and functionally diverged. This is supported by the close linkage to HoxC and HoxD which are assumed to be duplicate loci. In addition, the teleost fish paralogs are more similar to mammalian SLC11A2 and may result from a third, fish specific, genome duplication [51,52]. The synteny analysis performed in the 
current study strongly supports the latter, suggesting that the ancestor of all vertebrates had a single SLC11 gene that quadrupled as part of the $2 \mathrm{R}$ genome duplications. Two of the new genes were lost, while two were maintained in the ancestor of tetrapods and teleosts. In teleosts the slc11a2 clade expanded as the result of 3R to originate two isoforms, slc11a2- $\alpha$ and slc11a2- $\beta$, while the slc11a1 ortholog was lost (Figure 13). Unlike other teleosts [36], trout's slc11a2 homologs do not seem to result from $3 \mathrm{R}$ but rather a salmonid specific tetraploidization [45].

The overall constitutive expression pattern of sea bass slc11 transcripts resembles that of the mammalian SLC11A2 gene [24-26], but also of slc11a2 genes described in other teleost fishes [33,35,42,43,45]. Slc11a2- $\alpha$ was found to be ubiquitously expressed, but with a higher expression along the digestive tract, with a particularly high incidence in the mid and posterior sections of the intestine. This already gives some indications for its possible involvement in intestinal iron absorption in sea bass, since the Slc11a2 mediated uptake of iron and other food derived metals in teleosts occurs predominantly in the mid and posterior regions of the intestine, as previously described $[46,47]$. Slc11a2$\beta$ was also found to be ubiquitously expressed, although unlike slc11a2- $\alpha$, being abundant in the liver and in varying levels in all other tested tissues. No clear pattern was observed regarding the distribution of the four isoforms of slc11a2- $\beta$, although a prevalence of the $\beta 1$ and $\beta 2$ forms in most tested tissues was evident. Further analysis is needed to determine the exact function and contribution of each isoform. It is possible that they function in different subcellular compartments, with some of the forms more relevant in the immune response, while others may play a more prominent role in overall iron metabolism.

The in situ hybridization results in the liver, spleen, head kidney and sections of the intestine mostly reflect the measured constitutive expression. In the liver and spleen there is a clear prevalence of slc11a2- $\beta$, whereas in the

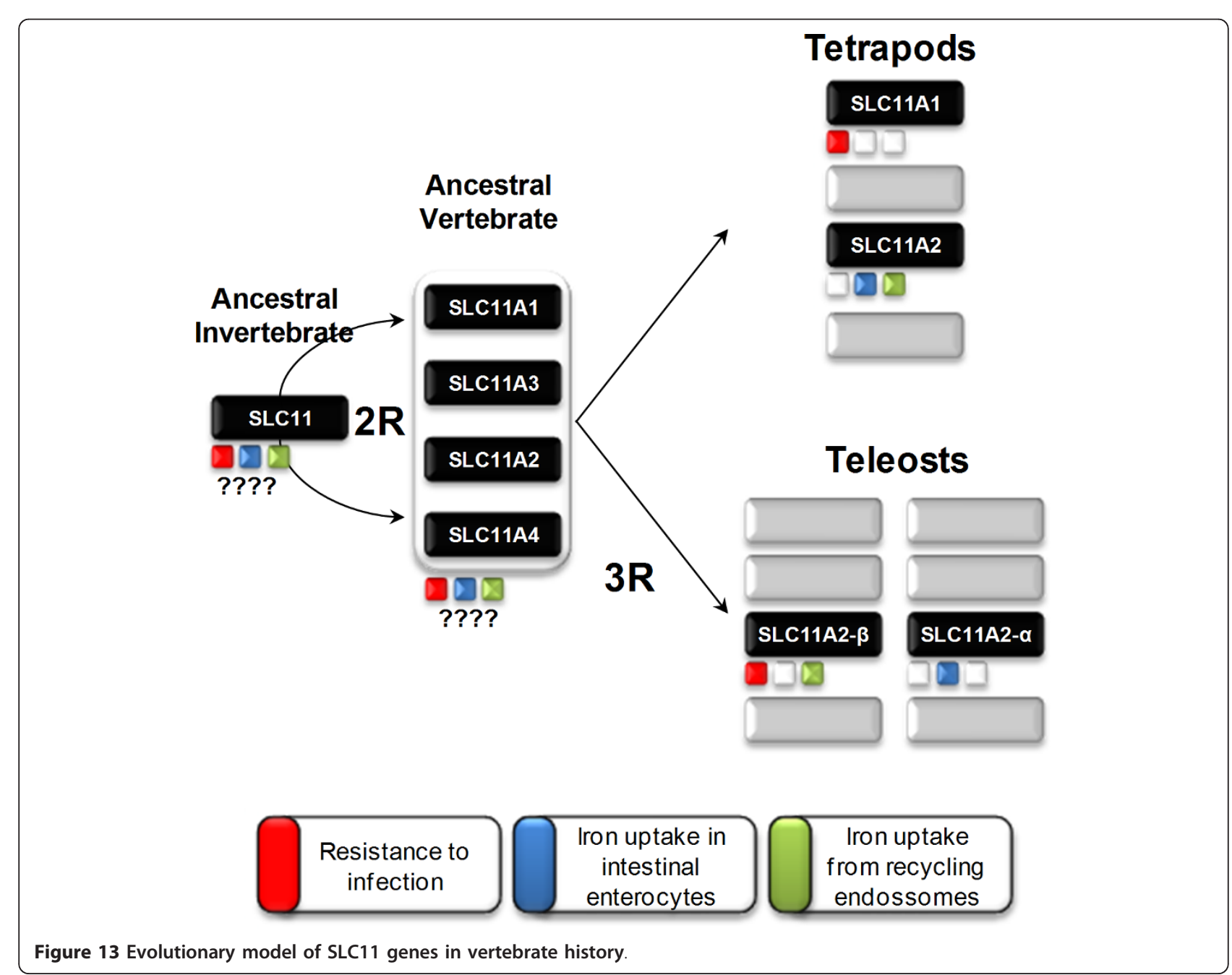


head kidney although the constitutive expression indicates a prevalence of slc11a2- $\beta$, there seems to be an almost even distribution of both forms. Note that in the spleen and kidney slc11a2- $\beta$ mRNA is clearly visible in the white pulp and lymphomyeloid tissue, respectively, which are both areas rich in B- and T-lymphocytes and actively involved in the immune response [75,76]. In the intestine, the in situ results show that slc11a2- $\alpha$ and slc11a2- $\beta$ are expressed in different compartments of the enterocytes, with slc11a2- $\alpha$ mRNA mostly concentrated close to the brush border of the apical pole, whereas slc11a2- $\beta$ is spread all over the cells, not only in the enterocytes but also in other intestinal cells. This observation provides further evidence for a sub-functionalization of slc11a2 homologs in sea bass, with slc11a2- $\alpha$ localization resembling that of the mammalian SLC11A2 IRE-positive isoforms and slc11a2- $\beta$ with the more general distribution of mammalian SLC11A2 IRE-negative isoforms [62,74,77]. An issue that remains puzzling is the distribution of slc11a2 isoforms in the posterior intestine, with both forms concentrated in or around the goblet cells, the intestinal glandular cells that secret mucin [78]. In mammals, there are conflicting reports on the presence of SLC11A2 in the goblet cells, with some reporting its absence [79] while others report its presence [80], suggesting that, along with mobilferrin, $S L C 11 A 2$ facilitates iron uptake.

To better understand the role of sea bass slc11a2 genes, in vivo and in vitro experimental models of iron modulation and infection were created. Haematological and iron parameters, and expression levels of slc11a2- $\alpha$ and slc11a2- $\beta$ were evaluated under those experimental conditions.

In the experimentally iron deficient animals, with decreased haematological parameters, slc11a2- $\alpha$ expression was significantly up-regulated in the intestine, whereas slc11a2- $\beta$ did not respond in any of the tested tissues. Further characterization of the slc11a2- $\alpha$ response to iron deficiency in the intestine showed that the up-regulation was mostly confined to the mid and posterior portions of the intestine. This is in accordance with previous findings that intestinal metal uptake in teleost fish occurs mainly in the posterior portion of the intestine [46,47], unlike mammals, where uptake occurs primarily in the duodenum [23]. These results further reinforce the idea that sea bass slc11a2- $\alpha$ seems to be mainly involved in iron uptake by the enterocytes.

In the experimentally iron overloaded animals, steady increases of the hematocrit and red blood cell counts were observed, as well as high levels of serum iron and transferrin saturation in the first days of the experiment. Also, increased levels of iron were observed in the liver, further confirming the iron overload status. Slc11a2- $\alpha$ expression did not change significantly in response to iron overload in any of the tested tissues and slc11a2- $\beta$ was up-regulated only in the liver. Together with the increased liver iron content, this is likely associated with the need to cope with the excess of iron introduced into the system, in order to prevent its toxic effects. In normal conditions, iron in the blood is bound to transferrin, and transferrin-iron complexes are internalized by a transferrin receptor-mediated endocytic pathway. Through acidification of the endosome, iron is released from transferrin and SLC11A2 is recruited to the endosome membrane, where it exports iron to the cytoplasm. Iron is then used in cellular processes or stored in ferritin molecules. When more iron is introduced into the system, an increased expression of these genes could be expected. We have already demonstrated that during iron overload there is an increased expression of ferritin, although not accompanied by an up-regulation of transferrin, most likely due the already high levels of constitutive expression in the liver [56].

In the in vivo experimental infection with live P. damselae, a significant and continuous decrease of all haematological parameters was observed, suggesting a condition that is commonly known as anaemia of inflammation or chronic disease. This response was first described in humans $[81,82]$ and later reported for teleost fish, namely sea bass $[56,57,59]$ and Nile tilapia [83]. During bacterial infection, inflammatory cytokines, such as IL-6, prompt the liver to increase the production of the iron metabolism regulatory peptide hepcidin, causing the internalization of ferroportin and thus preventing cellular iron export $[82,84]$. The iron retention leads to impairment in erythropoiesis, due to the lack of iron availability. Overall, these changes contribute to limiting the availability of iron for pathogen growth. We have previously shown that hepcidin [59] and ferritin [56] levels are increased during bacterial infection in sea bass, thus confirming the presence of this mechanism in teleosts.

The bacterial challenge significantly up-regulated the expression of slc11a2- $\beta$ in the liver, spleen and head kidney, in a time-dependent fashion, but produced no significant changes in slc11a2- $\alpha$ expression. A similar up-regulation was observed in slc11a2 homologs of other teleost fish in response to $V$. anguillarum [34,35], Mycobacterium [32], E. ictaluri [48] and LPS [43]. Further analysis will be required in order to determine whether in this case Slc11a2- $\beta$ is acting as the mammalian counterpart of SLC11A1, being recruited to the membrane of pathogen-containing phagosomes, or of SLC11A2, being recruited to the membrane of holotransferrin-positive recycling endosomes involved in iron uptake from the extracellular environment. Since there are no SLC11A1 homologs in sea bass, and there are 4 different proteins encoded by $\operatorname{slc} 11 a 2-\beta$, it is 
tempting to propose that Slc11a2- $\beta$ is performing both SLC11A1 and SLC11A2 mammalian functions.

In the in vitro iron overload of spleen-isolated leukocytes, slc11a2- $\beta$ was found to be up-regulated in a timedependent fashion, whereas no significant changes in slc11a2- $\alpha$ expression were observed. The increase in slc11a2- $\beta$ expression is most likely due to the increase in iron accumulation in the macrophages, to cope with the excess iron introduced in the medium, which could become toxic for the cells.

The in vitro experimental infection with heat-inactivated $P$. damselae, in spleen-isolated leukocytes, produced the same effect as that observed in vivo, with an up-regulation of slc11a2- $\beta$ and no changes in slc11a2- $\alpha$ expression. Not much information is available on the expression of SLC11 homologs in leukocytes, but it is known that mammalian SLC11A1 expression is stimulated by M. avium in peritoneal macrophages [85] and by LPS and IFN- $\gamma$ in RAW264.7 macrophages [86]. Mammalian SLC11A2 expression is also stimulated by $M$. avium in peritoneal macrophages, although differentially from SLC11A1 [85]. Also, catfish slc11 was found to be stimulated in monocytes by LPS [43]. As for the in vivo experimental infection, it has yet to be determined whether Slc11a2- $\beta$ is functioning as SLC11A1 or SLC11A2, but since both forms are known to be required for an efficient erythrophagocytosis and iron recycling in macrophages [87], it is likely that it is performing both functions. Expression studies of the four slc11a2- $\beta$ forms and subcellular localization in the leukocytes could provide some insight on this matter.

\section{Conclusions}

We have successfully isolated two slc11 paralogs in sea bass, that we named slc11a2- $\alpha$ and slc11a2- $\beta$. We have demonstrated that they have a role not only in iron metabolism but also in the response to bacterial infection. The clarification of the evolutionary scenario along with the functional data suggests a curious and complex pattern of sub-functionalization [88] and paralog functional equivalence, a process previously named as synfunctionalization $[89,90]$ : the fish specific paralog slc11a2- $\alpha$ retained in part the original mammalian SLC11A2 function, whereas the slc11a2- $\beta$ isoform not only retained the original $S L C 11 A 2$ function but also acquired the $S L C 11 A 1$ function after the loss of this isoform in teleosts (Figure 13).

\section{Methods}

\section{Fish rearing}

European sea bass (Dicentrarchus labrax), with an average weight of $50 \mathrm{~g}$, were provided by a commercial fish farm in the north of Portugal (Aquacircia, Aveiro, Portugal) and reared at the fish holding facilities of the
Centro Interdisciplinar de Investigação Marinha e Ambiental (CIIMAR, Porto, Portugal). Fish were maintained in 2000 liter tanks with water recirculation at a temperature of $12-14^{\circ} \mathrm{C}$ and constant salinity (32 ppm). The fish were fed daily to satiation with commercial fish feed (EWOS, West Lothian, UK) with an iron content of approximately $200 \mathrm{mg}$ iron $/ \mathrm{kg}$ feed and kept for more than three weeks prior to experimental use. At the beginning of each treatment fish were anaesthetized with $100 \mathrm{mg} / \mathrm{l}$ water of tricaine methanesulfonate (MS222) (Pharmaq, Fordingbridge, UK). All animal experiments were carried out in strict compliance with national and international animal use ethics guidelines, approved by the animal welfare and ethic committees of IBMC and CIIMAR (permit ref. Ofício Circular $n^{\circ} 99$, 0420/000/000 of 09/11/2009 from the Direcção Geral de Veterinária (DGV), Portuguese Ministry of Agriculture, Rural Development and Fisheries) and conducted by FELASA Category C/DGV certified investigators.

\section{RNA isolation and CDNA synthesis}

Fish were euthanized by anesthetic overdose of MS222 $(250 \mathrm{mg} / \mathrm{l})$, dissected and tissue samples collected, snap frozen in liquid nitrogen and stored at $-80^{\circ} \mathrm{C}$ until further use. Total RNA was isolated with the RNeasy Midi Kit for Total RNA Isolation from Animal Cells (Qiagen, Valencia CA, USA) with the optional On-Column DNase Digestion with RNase-free DNase (Qiagen). Total RNA quantification was performed using a NanoDrop 1000 spectrophotometer (Thermo Scientific, Waltham MA, USA), quality was assessed by visualization in a denaturating formaldehyde-agarose gel and $1.25 \mu \mathrm{g}$ of each sample were converted to cDNA by Thermoscript ${ }^{\mathrm{TM}}$ and an oligo (dT) 20 primer (Invitrogen, Carlsbad CA, USA), according to the manufacturer's protocol.

\section{Southern blot assay}

Genomic DNA was isolated from sea bass red blood cells, as described elsewhere [91]. To determine the number of slc11 gene copies in sea bass, $10 \mu \mathrm{g}$ of genomic DNA were independently digested for $24 \mathrm{~h}$ with EcoRI or HindII (Roche Applied Science, Mannhelm, Germany). Digestion products were run on an appropriate electrophoresis gel and blotted onto a positively charged nylon membrane (Byodine ${ }^{\circledR}$ Plus Membrane, Pall Life Sciences, Ann Arbor MI, USA). The membrane was subjected to Southern analysis using the DIG System (Roche Applied Science) according to the manufacturer's specifications. Briefly, a 152 bp slc11 DIG labeled probe was prepared with the DIG Probe Synthesis Kit (Roche Applied Science), using primers designed based on other fish slc11 mRNA sequences (supplementary table S1), the membrane was hybridized at $55^{\circ} \mathrm{C}$, washed, and detection was performed with the chemiluminescent substrate 
CDP-Star (Roche Applied Science). Visualization was achieved by exposing the membrane to X-ray film (Hyperfilm $^{\mathrm{TM}}$, GE healthcare, Buckinghamshire, England) for 5-20 minutes.

\section{Isolation of sea bass slc11 genes}

Several pairs of oligonucleotide PCR primers (Additional File 6, Table S1) were designed according to highly conserved regions of slc11a1 and slc11a2 mRNA sequences from other fish and mammalian species, available in the National Center for Biotechnology Information nucleotide database [92] and Ensembl [93] and cDNA preparations from liver, spleen and intestine were used in PCR amplifications. PCR products were run on $1.2 \%$ agarose gels, relevant fragments were purified with the QIAquick Gel Extraction Kit (Qiagen), cloned into pGEM-T Easy vectors, propagated in JM109 High Efficiency Competent Cells (Promega Corporation, Madison WI, USA) and sent for sequencing (Stabvida, Oeiras, Portugal).

The derived cDNA sequences were compared by megablast alignment with genomic contigs of an early version of the D. labrax whole genome shotgun assembly (Max Planck Institute for Molecular Genetics, Berlin, Germany). The genomic contigs were the result of an assembly of $\sim 3 \times$ genome coverage Sanger sequencing reads and $\sim 3 \times$ coverage 454 FLX Titanium pyro-sequencing reads by the Celera Assembler v5.3 [94]. Contigs that matched the cDNA sequences were used to build the gene models as implied by mRNA sequences.

\section{Rapid amplification of CDNA ends (5'and $3^{\prime}$ RACE)}

Both 5' and 3' RACE were carried out using the 5'/3' RACE Kit, $2^{\text {nd }}$ Generation (Roche Applied Science) according to the manufacturer's instructions. Conditions for PCR were: $94^{\circ} \mathrm{C}$ for $2 \mathrm{~min}, 94^{\circ} \mathrm{C}$ for $15 \mathrm{~s}, 60^{\circ} \mathrm{C}$ for $30 \mathrm{~s}, 72^{\circ} \mathrm{C}$ for $40 \mathrm{~s}$, for 10 cycles; $94^{\circ} \mathrm{C}$ for $15 \mathrm{~s}, 60^{\circ} \mathrm{C}$ for $30 \mathrm{~s}, 72^{\circ} \mathrm{C}$ for $40 \mathrm{~s}$ (plus $20 \mathrm{~s} /$ cycle), for 25 cycles, with a final elongation at $72^{\circ} \mathrm{C}$ for $7 \mathrm{~min}$. When necessary, a second PCR amplification was performed using these conditions for an additional 30 cycles. PCR products were run on $1.2 \%$ agarose gels, relevant fragments were purified with the QIAquick Gel Extraction Kit (Qiagen), cloned and sequenced as described earlier.

\section{Sequence analysis and alignment}

Both strands of the cDNA were sequenced and assembled using Multalin $[95,96]$ and by manual comparison of overlapping electropherograms. Alignments of the amino acid sequences of the Slc11 predicted proteins were performed using ClustalW from MEGA5 [97]. Phylogenetic tree was constructed using the Maximum-Likelihood method, with the Jones-Taylor-Thornton (JTT) model, Nearest-Neighbor-Interchange (NNI) heuristic model, complete deletion of gaps and 1000 bootstrap replications, with MEGA5 and PAUP* v4.0b10 [98]. Additional phylogenetic trees were constructed using Bayesian inference, with MrBayes v3.1.2 [99,100] and Maximum-Parsimony and Neighbor-Joining method of Saitou and Nei [101], with MEGA5. Sequences used for comparisons and phylogenetic trees and their accession numbers were as follows: from GenBank - striped bass Slc11 (AAG31225), turbot Slc11a2- $\beta$ (ABB73023), turbot Slc11a2- $\gamma$ (ABE97051), rainbow trout Slc11a2- $\alpha$ (AAD20721), rainbow trout Slc11a2- $\beta$ (AAD20722), fugu Slc11a2- $\alpha$ (CAD43050), fugu Slc11a2- $\beta$ (CAD4 3051), red seabream Slc11 (AAR83912), halibut Slc11 (AAX86980), channel catfish Slc11 (AAM73759), carp Slc11 (CAB60196), zebrafish Slc11 (NP_001035460), chicken SLC11A1 (NP_990295), mouse SLC11A1 (NP_ 038640), mouse SLC11A2 (NP_001139633), human SLC11A1 (NP_000569), human SLC11A2 -IRE (AAC2 1459), human SLC11A2 +IRE (NP_000608) and Drosophila Mvl-RA (NP_524425); from Ensembl - medaka Slc11a2- $\alpha$ (ENSORLP00000020758), medaka Slc11a2- $\beta$ (ENSORLP00000019423), stickleback Slc11a2- $\alpha$ (ENS GACP00000015490), stickleback Slc11a2- $\beta$ (ENSG ACP00000000618), tetraodon Slc11a2- $\beta$ (ENSTNIP 00000009880), Anole lizard Slc11a1 (ENSACAP0000 0001836), Anole lizard Slc11a2 (ENSACAP0000 0012437), Xenopus Slc11a1 (ENSXETP00000032694), Xenopus Slc11a2 (ENSXETP00000021232) and $C$. elegans SMF-1 (K11G12.4b).

\section{Genomic organization}

Genomic DNA was amplified by RT-PCR with the primers previously used for cDNA (Additional File 6, Table S1) and several PCR products were purified, cloned and sent for sequencing (Stabvida). Whole genome shotgun reads (Max Planck Institute for Molecular Genetics) were used to extend genomic regions that could not be amplified by RT-PCR. Comparisons were made between cDNA and genomic DNA to assess the similarity of the coding regions and to identify intron/exon boundaries.

Sea bass slc11 genes genomic organization was compared with the sequences for fugu (Takifugu rubripes) slc11a2, tetraodon (Tetraodon nigroviridis) slc11a2, xenopus (Xenopus tropicalis) slc11a1 and slc11a2, mouse (Mus musculus) Slc11a1 and Slc11a2, human (Homo sapiens) SLC11A1 and SLC11A2 and Drosophila (Drosophila melanogaster) $M v l-R A$, available at Ensembl, release 57.

\section{Paralogy and synteny analysis}

We identified and located the human orthologs of SLC11A1 (Hsa2q) and SLC11A2 (Hsa12q) using the Ensembl database release 57 . These regions are part of the Hox paralogon [36], along with Hsa17q and Hsa3p/7. To infer if the human SLC11A1 and SLC11A2 gene 
environment displayed signs of duplication attributable to the $2 \mathrm{R}$ genome duplications, the gene content in a $1 \mathrm{Mb}$ Ensembl window was analysed. Gene families with more members mapping to distinct regions of the Hox paralogon were identified. Their phylogenenic relationships were inferred from the phylogenies generated by the Ensembl orthology/paralogy pipeline. Synteny data for the teleost species was retrieved from Ensembl databases. Orthology/paralogy relationships and location of human sequences were derived from the ortholog/paralog prediction function of the Ensembl website. Phylogenetic relationship of the duplicated teleost genes was determined as previously outlined.

\section{Slc11a2- $\alpha$ and slc11a2- $\beta$ constitutive expression by real- time RT-PCR}

Relative levels of slc11a2- $\alpha$ and slc11a2- $\beta$ mRNAs were quantified by real-time PCR analysis using an iQ5 Multicolor Real-Time PCR Detection System (Bio-Rad, Hercules CA, USA). One $\mu$ of each cDNA sample was added to a reaction mix containing $10 \mu \mathrm{l}$ iQ SYBR Green Supermix (Bio-Rad), $8.5 \mu \mathrm{l}$ of ${ }_{\mathrm{dd}} \mathrm{H}_{2} \mathrm{O}$ and $250 \mathrm{nM}$ of each primer, making a total volume of $20 \mu \mathrm{l}$ per reaction. A non-template control was included for each set of primers (supplementary table S1). The cycling profile was the following: $94^{\circ} \mathrm{C}$ for $3.5 \mathrm{~min}, 40$ cycles of $94^{\circ} \mathrm{C}$ for $30 \mathrm{~s}, 59^{\circ} \mathrm{C}$ for $30 \mathrm{~s}$ and $72^{\circ} \mathrm{C}$ for $30 \mathrm{~s}$. A melting curve was generated for every PCR product to confirm the specificity of the assays and a dilution series was prepared to check the efficiency of the reactions. $\beta$-actin was used as the housekeeping gene. The comparative CT method ( $2^{-\Delta \Delta C T}$ method) based on cycle threshold (CT) values for slc11a2- $\alpha$, slc11a2- $\beta$ and $\beta$-actin was used to analyze the expression levels of slc11a2- $\alpha$ and slc11a2- $\beta$.

\section{Slc11a2- $\beta$ isoforms constitutive expression by semi- quantitative RT-PCR}

Since the different 5 ' and $3^{\prime}$ exons are separated by approximately $1.6 \mathrm{~kb}$, evaluation of the constitutive expression of the four isoforms of slc11a2- $\beta$ by realtime PCR was not a viable option. Hence, we optimized the conditions for semi-quantitative PCR to be able to reliably and specifically distinguish between these variants. To this end, exon 1A- or 1B-specific forward primers were combined with exon 15- or 16-specific reverse primers (Additional File 6, Table S1). PCRs were performed with the following conditions: 10-30 cycles of $94^{\circ} \mathrm{C}$ for $30 \mathrm{~s}, 59^{\circ} \mathrm{C}$ for $30 \mathrm{~s}$ and $72^{\circ} \mathrm{C}$ for $2 \mathrm{~min}$, with an initial $5 \mathrm{~min}$ denaturation at $94^{\circ} \mathrm{C}$ and a final $10 \mathrm{~min}$ extension at $72^{\circ} \mathrm{C}$. PCR products were resolved on ethidium bromide-stained $1.2 \%$ agarose gels, scanned on a GelDocXR+ (Biorad) and quantified using Quantity One software (Biorad).

\section{In situ hybridization}

All reagents used for in situ hybridization were prepared with $0.1 \%$ diethyl pyrocarbonate (DEPC) in double-distilled $\mathrm{H}_{2} \mathrm{O}$ to rid of RNases from working solutions. Digoxigenin (DIG)-labeled anti-sense and sense riboprobes for slc11a2- $\alpha$ and slc11a2- $\beta$ of approximately $150 \mathrm{bp}$ were synthesized in vitro from linearized plasmid DNA, following the DIG-UTP supplier instructions (Roche Applied Science). Sections of liver, spleen, head kidney and anterior $/ \mathrm{mid} /$ posterior regions of intestine were fixed in freshly prepared $4 \%$ paraformaldehyde in $100 \mathrm{mM}$ phosphate-buffered saline (PBS; $\mathrm{pH} 7.4$ ) at $4^{\circ} \mathrm{C}$ for $8 \mathrm{~h}$. After dehydration, sections were embedded in paraffin, sectioned at $3 \mu \mathrm{m}$, mounted on poly-L-lysine coated slides, and dried at $42^{\circ} \mathrm{C}$ for $36 \mathrm{~h}$. The sections were dewaxed in xylene 4 times ( 2 min each), followed by immersion in 100\% ethanol (1 min), 90\% ethanol (30 s), $75 \%$ ethanol (45 s) and washed with water (1 min). They were then pre-hybridized in a hybridization buffer containing $50 \%(\mathrm{v} / \mathrm{v})$ deionized formamide, $50 \mu \mathrm{g} / \mathrm{ml}$ heparin, $5 \times$ standard saline citrate (SSC), 0.1\% Tween-20, $9.2 \mathrm{mM}$ citric acid pH 6.0 and $0.5 \mathrm{mg} / \mathrm{ml}$ total yeast RNA at $70^{\circ} \mathrm{C}$ for $2 \mathrm{~h}$, and hybridized in the same hybridization buffer with $1 \mu \mathrm{g} / \mathrm{ml}$ of DIG-labeled anti-sense or sense riboprobes at $70^{\circ} \mathrm{C}$ for $12-16 \mathrm{~h}$ in a humidified chamber. Subsequently, the sections were subjected to several washes at $70^{\circ} \mathrm{C}$ : 10 min with $75 \%$ wash buffer $(65 \%$ (V/V) deionized formamide, $5 \times$ SSC and $0.1 \%$ Tween-20)/25\% $2 \times$ SSC, 10 min with $50 \%$ wash buffer $/ 50 \% 2 \times$ SSC, 10 min with $25 \%$ wash buffer $/ 75 \% 2 \times$ SSC, 10 min with $2 \times$ SSC and twice for at least $30 \mathrm{~min}$ with $0.05 \times$ SSC. These were followed by washes at room temperature, with shaking: $5 \mathrm{~min}$ in $50 \% 0.05 \times \mathrm{SSC} / 50 \%$ tris(hydroxymethyl)aminomethane$\mathrm{HCl}$ (Tris- $\mathrm{HCl})(\mathrm{pH} 7.4)$ with $150 \mathrm{mM} \mathrm{NaCl}$ and twice for 5 min with $100 \mathrm{mM}$ Tris- $\mathrm{HCl}$ (pH 7.4) with $150 \mathrm{mM}$ $\mathrm{NaCl}$. Slides were then pre-incubated in blocking reagent (2\% normal goat serum and $2 \mathrm{mg} / \mathrm{ml}$ of bovine serum albumin) in $100 \mathrm{mM}$ Tris- $\mathrm{HCl}$ (pH 7.4) with $150 \mathrm{mM}$ $\mathrm{NaCl}$ for $1 \mathrm{~h}$ at room temperature with shaking and incubated with anti-DIG alkaline phosphatase conjugated antibody (Roche Applied Science) diluted 1:100 in blocking reagent for $2 \mathrm{~h}$ at room temperature. The sections were washed three times (5 min each) in $100 \mathrm{mM}$ Tris- $\mathrm{HCl}(\mathrm{pH}$ 7.4) with $100 \mathrm{mM} \mathrm{NaCl}$ and $50 \mathrm{mM} \mathrm{MgCl}_{2}$, then incubated with a coloring solution consisting of $4.5 \mu \mathrm{g} / \mathrm{ml}$ $\mathrm{NBT}$ and $3.5 \mu \mathrm{g} / \mathrm{ml} \mathrm{BCIP}$ in $100 \mathrm{mM}$ Tris- $\mathrm{HCl}(\mathrm{pH} 8.0)$ with $100 \mathrm{mM} \mathrm{NaCl}$ and $50 \mathrm{mM} \mathrm{MgCl}_{2}$ for $2-24 \mathrm{~h}$ in the dark. The color reaction was stopped in PBS for $10 \mathrm{~min}$. After rinsing in distilled water, the sections were mounted with Faramount Mounting Medium (Dako, Carpinteria CA, USA) and photographed under an Olympus BH-2 microscope (Olympus, Tokyo, Japan), with an Olympus DP25 digital camera (Olympus). Control sections were 
also prepared and stained with hematoxylin and eosin (H\&E).

\section{In vivo model of experimental iron modulation}

In order to modulate the sea bass iron status, fish reared as described before were anaesthetized and subjected to one of three different treatments, forming three experimental groups: iron overload, iron deficient and control (15 fish per group). To induce iron overload, fish were intraperitoneally injected with $200 \mu$ of Iron Dextran (Sigma, St. Louis, MO, USA) diluted in sterile PBS to a final concentration of $10 \mathrm{mg} / \mathrm{ml}$, as previously reported [57]. To induce the iron deficient state, fish were bled from the caudal vessels (approximately $500 \mu$ of blood). Control fish were injected with $200 \mu \mathrm{l}$ of sterile PBS. Four, seven and fourteen days after treatment, 5 fish from each of the experimental groups were anaesthetized and blood was drawn from the caudal vessels for evaluation of hematological parameters. Subsequently, fish were euthanized with an overdose of anesthetic, dissected and several tissues excised, snap frozen in liquid nitrogen and stored at $-80^{\circ} \mathrm{C}$ for further iron content evaluation and gene expression analysis.

\section{In vivo model of experimental infection}

Photobacterium damselae spp. piscicida, strain DI21, known to be pathogenic in sea bass, was used for the experimental infections. P. damselae was cultured to mid-logarithmic growth in tryptic soy broth (TSB) growth medium, supplemented with $1 \% \mathrm{NaCl}$. After measuring absorbance at $600 \mathrm{~nm}$, bacteria were resuspended in TSB $1 \% \mathrm{NaCl}$ to a final concentration of $5.0 \times 10^{5}$ CFUs $\mathrm{ml}^{-1}$.

For the experimental infection, 48 fish were anaesthetized and intraperitoneally injected with $200 \mu \mathrm{l}\left(1.0 \times 10^{5}\right.$ $\mathrm{CFU}$ ) of bacterial suspension. For the control group, 25 fish were injected with $200 \mu \mathrm{l}$ of TSB $1 \% \mathrm{NaCl}$. At 24, 48, 72 and $96 \mathrm{~h}$ of infection, 6 fish from each group were anaesthetized and blood drawn from the caudal vessels for evaluation of hematological parameters. Fish were then euthanized with an overdose of anesthetic, dissected and several tissues excised, snap frozen in liquid nitrogen and stored at $-80^{\circ} \mathrm{C}$ for further iron content evaluation and gene expression analysis. Mortality was assessed every $12 \mathrm{~h}$ during the experimental infection.

\section{Hematological parameters and tissue iron in the in vivo experimental models}

In order to determine hematological parameters, peripheral blood was drawn from the caudal vessels. For red blood cells count and hematocrit determination, $150 \mu \mathrm{l}$ of blood were used in a 1:1 dilution with heparin in PBS (1000 units/ml). For determination of serum iron, non- heparinized blood was transferred into $1.5 \mathrm{ml}$ microcentrifuge tubes, and allowed to clot for $12 \mathrm{~h}$ at $4^{\circ} \mathrm{C}$. The samples were centrifuged twice at $16000 \times$ g until a clear serum was obtained. Serum iron (SI), unsaturated iron binding capacity (UIBC), total iron binding capacity (TIBC) and transferrin saturation (TS) were determined by the liquid ferrozine ${ }^{\circledR}$ method (Thermo Electron, Victoria, Australia) according to the manufacturer's specifications.

Non-heme iron was measured in livers by the bathophenanthroline method [102]. Briefly, liver samples were weighted, placed in iron-free Teflon vessels (ACVAdvanced Composite Vessel, CEM Corporation, Matthews NC, USA) and dried in a microwave oven (MDS 2000, CEM Corporation). Subsequently, dry tissue weights were determined and samples digested in an acid mixture (30\% hydrochloric acid and 10\% trichloroacetic acid) for $20 \mathrm{~h}$ at $65^{\circ} \mathrm{C}$. After digestion, a chromogen reagent (5 volumes of deionised water, 5 volumes of saturated sodium acetate and 1 volume of $0.1 \%$ bathophenanthroline sulfonate/ $1 \%$ thioglycollic acid) was added to the samples in order to react with iron and obtain a colored product that was measured spectrophotometrically at $535 \mathrm{~nm}$. The extinction coefficient for bathophenanthroline is $22.14 \mathrm{mM}^{-1} \mathrm{~cm}^{-1}$.

\section{In vitro experimental models of iron overload and infection}

Leucocytes were isolated from sea bass spleens. For each experiment (infection and iron overload), 5 untreated healthy fish were anesthetized and bled from the caudal vessels, then euthanized with anesthetic overdose. Spleens were aseptically dissected, placed in isolation medium (RPMI with 0.1\% Fetal Bovine Serum (FBS), 1\% Essential Amino Acids (A/A), 1\% MEM Non-essential Amino Acid solution (MEMNEAA), $0.35 \% \mathrm{NaCl} 1 \mathrm{M}$ and $0.4 \%$ heparin) for $10 \mathrm{~min}$ and macerated, with the addition of isolation medium, over a $0.4 \mu \mathrm{m}$ mesh into a 15 $\mathrm{ml}$ centrifuge tube. Volumes were adjusted to $10 \mathrm{ml}$ and centrifuged at $400 \times \mathrm{g}$ for $10 \mathrm{~min}$, at $4^{\circ} \mathrm{C}$. The pellet was resuspended in $5 \mathrm{ml}$ of isolation medium, overlaid on 5 $\mathrm{ml}$ of Lymphoprep (Axis-Shield PoC AS, Oslo, Norway) and centrifuged at $800 \times \mathrm{g}$ for $30 \mathrm{~min}$, at $4^{\circ} \mathrm{C}$. Cells were collected from the interface and washed twice with isolation medium at $1000 \times \mathrm{g}$ for $10 \mathrm{~min}$. The pellets were resuspended in complete culture medium (RPMI with 5\% FBS, $1 \%$ A/A, $1 \%$ MEMNEAA, $5 \%$ SBS and $0.35 \% \mathrm{NaCl}$ ) to a final concentration of $2.5 \times 10^{6}$ cells $/ \mathrm{ml}$.

For the iron overload experiment, cells were distributed between twelve 6-well flat bottom plates (Sarstedt, Nümbrecht, Germany), 5 fish per plate, $2 \mathrm{ml}$ per well $\left(5 \times 10^{6}\right.$ cells). The control and iron overload groups ( 6 plates each) received $200 \mu \mathrm{l}$ of $0.1 \mathrm{M}$ ammonium citrate (Sigma) or $200 \mu \mathrm{l}$ of $0.1 \mathrm{M}$ ferric ammonium citrate (Sigma, iron 
content of 16.5-18.5\%) per well, respectively. The latter is equivalent to approximately $8 \mu \mathrm{g}$ of iron per well. For the experimental infection, cells were distributed as previously described and the control and infected groups (6 plates each) received $200 \mu \mathrm{l}$ of PBS or $200 \mu \mathrm{l}$ of heat-inactivated Photobacterium damselae $\left(1 \times 10^{8}\right.$ cells $\left./ \mathrm{ml}\right)$ per well, respectively. Plates were kept in an incubator at $23^{\circ} \mathrm{C}$ for the duration of the experiments.

At $0,6,12,24,48$ and 72 hours post-treatments, cells were collected and RNA was isolated. Briefly, cells in suspension were collected into RNase-free tubes and $0.2 \%$ trypsin in PBS was added to the wells until adherent cells detached. Complete culture medium was added and the cell suspension was transferred into the same RNase-free tubes. RNA was isolated according to the RNeasy Plus Mini protocol (Qiagen) and cDNA was prepared with Thermoscript (Invitrogen) as described before.

\section{SIc11a2- $\alpha$ and s/c11a2- $\beta$ expression under iron modulation and infection in the in vivo and in vitro models}

The relative levels of slc11a2- $\alpha$ and slc11a2- $\beta$ mRNAs in the organs (in vivo models) and leukocytes (in vitro models) were quantified by real-time RT-PCR. Total RNA isolation, cDNA preparation and real-time RTPCR were performed as described before.

\section{Statistical analysis}

Statistical analysis was carried out using PASW Statistics v17.0 for Windows (SPSS Inc., Chicago IL, USA). Data normality was checked by performing KolmogorofSmirnoff test and Student's T-test was used for estimating statistical significance. Multiple comparisons were performed with ANOVA. A p value less than 0.05 was considered statistically significant.

\section{Additional material}

Additional File 1: Figure S1: DNA and predicted amino acid sequence of sea bass slc11a2 isoforms. This file contains the cDNA sequences, as well as the putative proteins and characteristic features for each slc11a2 isoform.

Additional file 2: Figure S2: Comparative view of the genomic structure, organization and size of SLC11 homologs of several species. This file contains a comparative view of the genomic structure and size of sea bass s/c11a2- $\alpha$ and s/c11a2- $\beta$ with homologs from other fishes, amphibians, mammals and insects.

Additional file 3: Figure S3: Additional phylogenetic trees. This file contains additional phylogenetic trees constructed with Bayesian, neighbour-joining and maximum-parsimony methods.

Additional file 4: Figure S4: Evolutionary relationships of AGAP2, ANKRD52 and MARCH9 gene families. This file contains phylogenetic trees for AGAP2, ANKRD52 and MARCH9, constructed with the maximum-likelihood method.
Additional file 5: Figure S5: Sea bass mortality during experimental infection. This file contains a graphic showing sea bass mortality during infection with Photobacterium damselae, at 12 hour intervals.

Additional file 6: Table S1: Primers used in this study. This file contains a table with all the primers used for sequencing, southern blot, in situ hybridization and gene expression analysis performed in this study.

\section{Acknowledgements}

The authors would like to thank Carolina Caldas, at IBMC, for technical assistance and support, Bernardo Balseiro, at Aquacircia, for donating the fish used in all the experiments and Dr. Salomé Gomes, Dr. Tiago Duarte, Dr. João Cabral and Dr. Jorge Vieira at IBMC for helpful discussions and comments on the manuscript. This work was supported by the Portuguese Fundação para a Ciência e a Tecnologia (FCT), grants PTDC/CVT/100386/ 2008 and SFRH/BD/29203/2006.

\section{Author details}

${ }^{1}$ Iron and Innate Immunity, Instituto de Biologia Molecular e Celular (IBMC), Rua do Campo Alegre 823, 4150-180 Porto, Portugal. ${ }^{2}$ Centro Interdisciplinar de Investigação Marinha e Ambiental (CIIMAR), Rua dos Bragas 289, 4050123 Porto, Portugal. ${ }^{3}$ Max-Planck-Institute for Molecular Genetics, Ihnestraße 63-73, 14195 Berlin, Germany. ${ }^{4}$ Instituto de Ciências Biomédicas Abel Salazar (ICBAS), Universidade do Porto, Largo Prof. Abel Salazar 2, 4099-003 Porto, Portugal.

\section{Authors' contributions}

JVN, JMW, PNSR conceived and designed the experiments, JVN, PNSR performed the experiments, JVN, JMW, LFCC, PNSR analysed the data, JVN, JMW, HK, RR, LFCC, PNSR contributed with reagents, materials and analysis tools, JVN, JMW, LFCC, PNSR prepared the manuscript. All authors read and approved the final manuscript.

\section{Competing interests}

The authors declare that they have no competing interests.

Received: 7 December 2010 Accepted: 18 April 2011

Published: 18 April 2011

\section{References}

1. Forbes JR, Gros P: Divalent-metal transport by NRAMP proteins at the interface of host-pathogen interactions. Trends Microbiol 2001, 9:397-403.

2. Forbes JR, Gros P: Iron, manganese, and cobalt transport by Nramp1 (Slc11a1) and Nramp2 (Slc11a2) expressed at the plasma membrane. Blood 2003, 102:1884-1892.

3. Goswami T, Bhattacharjee A, Babal P, Searle S, Moore E, Li M, Blackwell JM: Natural-resistance-associated macrophage protein 1 is an $\mathrm{H}+$ /bivalent cation antiporter. Biochem J 2001, 354:511-519.

4. Techau ME, Valdez-Taubas J, Popoff JF, Francis R, Seaman M, Blackwell JM: Evolution of differences in transport function in SIc11a family members. J Biol Chem 2007, 282:35646-35656.

5. Gruenheid S, Pinner E, Desjardins M, Gros P: Natural resistance to infection with intracellular pathogens: the Nramp1 protein is recruited to the membrane of the phagosome. J Exp Med 1997, 185:717-730.

6. Searle S, Bright NA, Roach TI, Atkinson PG, Barton CH, Meloen RH, Blackwell JM: Localisation of Nramp1 in macrophages: modulation with activation and infection. J Cell Sci 1998, 111((Pt 19)):2855-2866.

7. Evans CA, Harbuz MS, Ostenfeld T, Norrish A, Blackwell JM: Nramp1 is expressed in neurons and is associated with behavioural and immune responses to stress. Neurogenetics 2001, 3:69-78.

8. Skamene E, Schurr E, Gros P: Infection genomics: Nramp1 as a major determinant of natural resistance to intracellular infections. Annu Rev Med 1998, 49:275-287.

9. Vidal SM, Malo D, Vogan K, Skamene E, Gros P: Natural resistance to infection with intracellular parasites: isolation of a candidate for Bcg. Cell 1993, 73:469-485. 
10. Gomes MS, Appelberg R: Evidence for a link between iron metabolism and Nramp1 gene function in innate resistance against Mycobacterium avium. Immunology 1998, 95:165-168.

11. Abel L, Sanchez FO, Oberti J, Thuc NV, Hoa LV, Lap VD, Skamene E, Lagrange PH, Schurr E: Susceptibility to leprosy is linked to the human NRAMP1 gene. J Infect Dis 1998, 177:133-145.

12. Bucheton B, Abel L, Kheir MM, Mirgani A, El-Safi SH, Chevillard C, Dessein A: Genetic control of visceral leishmaniasis in a Sudanese population: candidate gene testing indicates a linkage to the NRAMP1 region. Genes Immun 2003, 4:104-109.

13. Hatta M, Ratnawati, Tanaka M, Ito J, Shirakawa T, Kawabata M: NRAMP1/ SLC11A1 gene polymorphisms and host susceptibility to Mycobacterium tuberculosis and M. leprae in South Sulawesi, Indonesia. Southeast Asian J Trop Med Public Health 2010, 41:386-394.

14. Tanaka G, Shojima J, Matsushita I, Nagai H, Kurashima A, Nakata K, Toyota E, Kobayashi N, Kudo K, Keicho N: Pulmonary Mycobacterium avium complex infection: association with NRAMP1 polymorphisms. Eur Respir J 2007, 30:90-96.

15. Sechi LA, Gazouli M, Sieswerda LE, Molicotti P, Ahmed N, Ikonomopoulos J, Scanu AM, Paccagnini D, Zanetti S: Relationship between Crohn's disease, infection with Mycobacterium avium subspecies paratuberculosis and SLC11A1 gene polymorphisms in Sardinian patients. World J Gastroenterol 2006, 12:7161-7164.

16. Esposito L, Hill NJ, Pritchard LE, Cucca F, Muxworthy C, Merriman ME, Wilson A, Julier C, Delepine M, Tuomilehto J, et al: Genetic analysis of chromosome 2 in type 1 diabetes: analysis of putative loci IDDM7, IDDM12, and IDDM13 and candidate genes NRAMP1 and IA-2 and the interleukin-1 gene cluster. IMDIAB Group. Diabetes 1998, 47:1797-1799.

17. Sanjeevi CB, Miller EN, Dabadghao P, Rumba I, Shtauvere A, Denisova A, Clayton D, Blackwell JM: Polymorphism at NRAMP1 and D2S1471 loci associated with juvenile rheumatoid arthritis. Arthritis Rheum 2000, 43:1397-1404.

18. Singal DP, Li J, Zhu Y, Zhang G: NRAMP1 gene polymorphisms in patients with rheumatoid arthritis. Tissue Antigens 2000, 55:44-47.

19. Gazouli M, Sechi L, Paccagnini D, Sotgiu S, Arru G, Nasioulas G, Vassilopoulos D: NRAMP1 polymorphism and viral factors in Sardinian multiple sclerosis patients. Can J Neurol Sci 2008, 35:491-494.

20. Ates O, Musellim B, Ongen G, Topal-Sarikaya A: NRAMP1 (SLC11A1): a plausible candidate gene for systemic sclerosis (SSc) with interstitial lung involvement. J Clin Immunol 2008, 28:73-77.

21. Bellamy R: Susceptibility to mycobacterial infections: the importance of host genetics. Genes Immun 2003, 4:4-11.

22. Awomoyi AA: The human solute carrier family 11 member 1 protein (SLC11A1): linking infections, autoimmunity and cancer? FEMS Immunol Med Microbiol 2007, 49:324-329.

23. Gunshin H, Mackenzie B, Berger UV, Gunshin Y, Romero MF, Boron WF, Nussberger S, Gollan JL, Hediger MA: Cloning and characterization of a mammalian proton-coupled metal-ion transporter. Nature 1997, 388:482-488.

24. Gruenheid S, Cellier M, Vidal S, Gros P: Identification and characterization of a second mouse Nramp gene. Genomics 1995, 25:514-525.

25. Tabuchi M, Yoshimori T, Yamaguchi K, Yoshida T, Kishi F: Human NRAMP2/ DMT1, which mediates iron transport across endosomal membranes, is localized to late endosomes and lysosomes in HEp-2 cells. J Biol Chem 2000, 275:22220-22228.

26. Vidal S, Belouchi AM, Cellier M, Beatty B, Gros P: Cloning and characterization of a second human NRAMP gene on chromosome 12q13. Mamm Genome 1995, 6:224-230.

27. Fleming MD, Romano MA, Su MA, Garrick LM, Garrick MD, Andrews NC: Nramp2 is mutated in the anemic Belgrade (b) rat: evidence of a role for Nramp2 in endosomal iron transport. Proc Natl Acad Sci USA 1998, 95:1148-1153.

28. Fleming MD, Trenor CC, Su MA, Foernzler D, Beier DR, Dietrich WF, Andrews NC: Microcytic anaemia mice have a mutation in Nramp2, a candidate iron transporter gene. Nat Genet 1997, 16:383-386.

29. Cellier M, Govoni G, Vidal S, Kwan T, Groulx N, Liu J, Sanchez F, Skamene E, Schurr E, Gros P: Human natural resistance-associated macrophage protein: cDNA cloning, chromosomal mapping, genomic organization, and tissue-specific expression. J Exp Med 1994, 180:1741-1752.

30. Kishi F: Isolation and characterization of human Nramp cDNA. Biochem Biophys Res Commun 1994, 204:1074-1080.
31. Hu J, Bumstead N, Burke D, Ponce de Leon FA, Skamene E, Gros P, Malo D: Genetic and physical mapping of the natural resistance-associated macrophage protein 1 (NRAMP1) in chicken. Mamm Genome 1995, 6:809-815.

32. Burge EJ, Gauthier DT, Ottinger CA, Van Veld PA: Mycobacterium-inducible Nramp in striped bass (Morone saxatilis). Infect Immun 2004, 72:1626-1636.

33. Chen SL, Wang ZJ, Xu MY, Gui JF: Molecular identification and expression analysis of natural resistance associated macrophage protein (Nramp) cDNA from Japanese flounder (Paralichthys olivaceus). Fish Shellfish Immunol 2006, 20:365-373.

34. Chen SL, Xu MY, Ji XS, Yu GC: Cloning and characterisation of natural resistance associated macrophage protein (Nramp) cDNA from red sea bream (Pagrus major). Fish Shellfish Immunol 2004, 17:305-313.

35. Chen SL, Zhang YX, Xu JY, Meng L, Sha ZX, Ren GC: Molecular cloning, characterization and expression analysis of natural resistance associated macrophage protein (Nramp) cDNA from turbot (Scophthalmus maximus). Comp Biochem Physiol B Biochem Mol Biol 2007, 147:29-37.

36. Sibthorpe D, Baker AM, Gilmartin BJ, Blackwell JM, White JK: Comparative analysis of two slc11 (Nramp) loci in Takifugu rubripes. DNA Cell Biol 2004, 23:45-58.

37. Rodrigues V, Cheah PY, Ray K, Chia W: malvolio, the Drosophila homologue of mouse NRAMP-1 (Bcg), is expressed in macrophages and in the nervous system and is required for normal taste behaviour. EMBO J 1995, 14:3007-3020

38. Consortium TCeS: Genome Sequence of the Nematode C. elegans: A Platform for Investigating Biology. Science 1998, 282:2012-2018.

39. Belouchi A, Cellier M, Kwan T, Saini HS, Leroux G, Gros P: The macrophagespecific membrane protein Nramp controlling natural resistance to infections in mice has homologues expressed in the root system of plants. Plant Mol Biol 1995, 29:1181-1196.

40. Portnoy ME, Liu XF, Culotta VC: Saccharomyces cerevisiae expresses three functionally distinct homologues of the nramp family of metal transporters. Mol Cell Biol 2000, 20:7893-7902.

41. Makui H, Roig E, Cole ST, Helmann JD, Gros P, Cellier MF: Identification of the Escherichia coli K-12 Nramp orthologue ( $\mathrm{MntH})$ as a selective divalent metal ion transporter. Mol Microbiol 2000, 35:1065-1078.

42. Saeij JP, Wiegertjes GF, Stet RJ: Identification and characterization of a fish natural resistance-associated macrophage protein (NRAMP) CDNA. Immunogenetics 1999, 50:60-66.

43. Chen H, Waldbieser GC, Rice CD, Elibol B, Wolters WR, Hanson LA: Isolation and characterization of channel catfish natural resistance associated macrophage protein gene. Dev Comp Immunol 2002, 26:517-531.

44. Donovan A, Brownlie A, Dorschner MO, Zhou Y, Pratt SJ, Paw BH, Phillips RB, Thisse C, Thisse B, Zon LI: The zebrafish mutant gene chardonnay (cdy) encodes divalent metal transporter 1 (DMT1). Blood 2002, 100:4655-4659.

45. Dorschner MO, Phillips RB: Comparative analysis of two Nramp loci from rainbow trout. DNA Cell Biol 1999, 18:573-583.

46. Bury N, Grosell M: Iron acquisition by teleost fish. Comp Biochem Physiol C Toxicol Pharmacol 2003, 135:97-105.

47. Bury NR, Walker PA, Glover CN: Nutritive metal uptake in teleost fish. $J$ Exp Biol 2003, 206:11-23.

48. Elibol-Flemming B, Waldbieser GC, Wolters WR, Boyle CR, Hanson LA: Expression analysis of selected immune-relevant genes in channel catfish during Edwardsiella ictaluri infection. J Aquat Anim Health 2009, 21:23-35.

49. Holland PW, Garcia-Fernandez J, Williams NA, Sidow A: Gene duplications and the origins of vertebrate development. Dev Supp/ 1994, 125-133.

50. Putnam NH, Butts $T$, Ferrier DE, Furlong RF, Hellsten U, Kawashima T, Robinson-Rechavi M, Shoguchi E, Terry A, Yu JK, et al: The amphioxus genome and the evolution of the chordate karyotype. Nature 2008, 453:1064-1071.

51. Taylor JS, Van de Peer Y, Braasch I, Meyer A: Comparative genomics provides evidence for an ancient genome duplication event in fish. Philos Trans R Soc Lond B Biol Sci 2001, 356:1661-1679.

52. Taylor JS, Van de Peer $Y$, Meyer A: Revisiting recent challenges to the ancient fish-specific genome duplication hypothesis. Curr Biol 2001, 11: R1005-1008. 
53. Chistiakov DA, Hellemans B, Volckaert FA: Review on the immunology of European sea bass Dicentrarchus labrax. Vet Immunol Immunopathol 2007, 117:1-16.

54. Kuhl H, Beck A, Wozniak G, Canario AV, Volckaert FA, Reinhardt R: The European sea bass Dicentrarchus labrax genome puzzle: comparative BAC-mapping and low coverage shotgun sequencing. BMC Genomics 2010, 11:68.

55. Negrisolo E, Kuhl H, Forcato C, Vitulo N, Reinhardt R, Patarnello T, Bargelloni L: Different Phylogenomic Approaches to Resolve the Evolutionary Relationships among Model Fish Species. Mol Biol Evol 2010, 27:2757-2774

56. Neves JV, Wilson JM, Rodrigues PN: Transferrin and ferritin response to bacterial infection: the role of the liver and brain in fish. Dev Comp Immunol 2009, 33:848-857.

57. Rodrigues PN, Pereira FA: A model for acute iron overload in sea bass (Dicentrarchus labrax L.). Lab Anim 2004, 38:418-424.

58. Rodrigues PN, Pereira FA: Effect of dietary iron overload on Photobacterium damselae ssp. piscicida pathogenicity in sea bass, Dicentrarchus labrax (L.). J Fish Dis 2004, 27:673-676.

59. Rodrigues PN, Vazquez-Dorado S, Neves JV, Wilson JM: Dual function of fish hepcidin: response to experimental iron overload and bacterial infection in sea bass (Dicentrarchus labrax). Dev Comp Immunol 2006, 30:1156-1167.

60. Zanuy S, Carrillo M, Felip A, Rodríguez L, Blázquez M, Ramos J, Piferrer F: Genetic, hormonal and environmental approaches for the control of reproduction in the European sea bass (Dicentrarchus labrax L.). Aquaculture 2001, 202:187-203.

61. Wiegertjes GF, Stet RJ, Parmentier HK, van Muiswinkel WB: Immunogenetics of disease resistance in fish: a comparative approach. Dev Comp Immunol 1996, 20:365-381.

62. Hubert N, Hentze MW: Previously uncharacterized isoforms of divalent metal transporter (DMT)-1: implications for regulation and cellular function. Proc Natl Acad Sci USA 2002, 99:12345-12350

63. Lee PL, Gelbart T, West C, Halloran C, Beutler E: The human Nramp2 gene: characterization of the gene structure, alternative splicing, promoter region and polymorphisms. Blood Cells Mol Dis 1998, 24:199-215.

64. Alternative Splice Site Predictor. [http://www.es.embnet.org/ mwang/assp. html].

65. Wang M, Marin A: Characterization and prediction of alternative splice sites. Gene 2006, 366:219-227.

66. RegRNA. [http://regrna.mbc.nctu.edu.tw/index.php]

67. Huang $\mathrm{HY}$, Chien $\mathrm{CH}$, Jen $\mathrm{KH}$, Huang HD: RegRNA: an integrated web server for identifying regulatory RNA motifs and elements. Nucleic Acids Res 2006, 34:W429-434.

68. ExPASy. [http://www.expasy.org/tools/].

69. Cellier M, Prive G, Belouchi A, Kwan T, Rodrigues V, Chia W, Gros P: Nramp defines a family of membrane proteins. Proc Natl Acad Sci USA 1995, 92:10089-10093.

70. Dreborg S, Sundstrom G, Larsson TA, Larhammar D: Evolution of vertebrate opioid receptors. Proc Natl Acad Sci USA 2008, 105:15487-15492.

71. Jaillon O, Aury JM, Brunet F, Petit JL, Stange-Thomann N, Mauceli E, Bouneau L, Fischer C, Ozouf-Costaz C, Bernot A, et al: Genome duplication in the teleost fish Tetraodon nigroviridis reveals the early vertebrate proto-karyotype. Nature 2004, 431:946-957.

72. Eisenstein RS: Iron regulatory proteins and the molecular control of mammalian iron metabolism. Annu Rev Nutr 2000, 20:627-662.

73. Hentze MW, Muckenthaler MU, Andrews NC: Balancing acts: molecular control of mammalian iron metabolism. Cell 2004, 117:285-297.

74. Tabuchi M, Tanaka N, Nishida-Kitayama J, Ohno H, Kishi F: Alternative splicing regulates the subcellular localization of divalent metal transporter 1 isoforms. Mol Biol Cell 2002, 13:4371-4387.

75. Cesta MF: Normal structure, function, and histology of the spleen. Toxicol Pathol 2006, 34:455-465.

76. Zapata AG, Chibá A, Varas A: 1 Cells and Tissues of the Immune System of Fish. In Fish Physiology. Volume. Volume 15. Edited by: George I, Teruyuki N. Academic Press; 1997:1-62.

77. Lam-Yuk-Tseung S, Gros P: Distinct targeting and recycling properties of two isoforms of the iron transporter DMT1 (NRAMP2, SIC11A2). Biochemistry 2006, 45:2294-2301.

78. Specian RD, Oliver MG: Functional biology of intestinal goblet cells. Am J Physiol 1991, 260:C183-193.
79. Canonne-Hergaux F, Gruenheid S, Ponka P, Gros P: Cellular and subcellular localization of the Nramp2 iron transporter in the intestinal brush border and regulation by dietary iron. Blood 1999, 93:4406-4417.

80. Conrad ME, Umbreit JN: Pathways of iron absorption. Blood Cells Mol Dis 2002, 29:336-355.

81. Cartwright GE, Wintrobe MM: The anemia of infection. XVII. A review. AdV Intern Med 1952, 5:165-226.

82. Andrews NC: Anemia of inflammation: the cytokine-hepcidin link. J Clin Invest 2004, 113:1251-1253.

83. Chen CY, Wooster GA, Bowser PR: Comparative blood chemistry and histopathology of tilapia infected with Vibrio vulnificus or Streptococcus iniae or exposed to carbon tetrachloride, gentamicin, or copper sulfate. Aquaculture 2004, 239:421-443.

84. Nemeth E, Rivera S, Gabayan V, Keller C, Taudorf S, Pedersen BK, Ganz T: IL6 mediates hypoferremia of inflammation by inducing the synthesis of the iron regulatory hormone hepcidin. J Clin Invest 2004, 113:1271-1276.

85. Zhong W, Lafuse WP, Zwilling BS: Infection with Mycobacterium avium differentially regulates the expression of iron transport protein mRNA in murine peritoneal macrophages. Infect Immun 2001, 69:6618-6624.

86. Govoni G, Canonne-Hergaux F, Pfeifer CG, Marcus SL, Mills SD, Hackam DJ, Grinstein S, Malo D, Finlay BB, Gros P: Functional expression of Nramp1 in vitro in the murine macrophage line RAW264.7. Infect Immun 1999, 67:2225-2232

87. Soe-Lin S, Apte SS, Mikhael MR, Kayembe LK, Nie G, Ponka P: Both Nramp1 and DMT1 are necessary for efficient macrophage iron recycling. Exp Hematol 2010, 38:609-617.

88. Force A, Lynch M, Pickett FB, Amores A, Yan YL, Postlethwait J: Preservation of duplicate genes by complementary, degenerative mutations. Genetics 1999, 151:1531-1545.

89. Gitelman I: Evolution of the vertebrate twist family and synfunctionalization: a mechanism for differential gene loss through merging of expression domains. Mol Biol Evol 2007, 24:1912-1925.

90. Liberles DA: Gene duplication: red queens, linkage, redundancy and synfunctionalization. Heredity 2008, 101:299-300.

91. Cummings SA, Thorgaard GH: Extraction of DNA from fish blood and sperm. Biotechniques 1994, 17, 426, 428, 430.

92. National Center for Biotechnology Information. [http://www.ncbi.nIm.nih. gov].

93. Ensembl. [http://www.ensembl.org]

94. Miller JR, Delcher AL, Koren S, Venter E, Walenz BP, Brownley A, Johnson J, Li K, Mobarry C, Sutton G: Aggressive assembly of pyrosequencing reads with mates. Bioinformatics 2008, 24:2818-2824.

95. Multalin. [http://bioinfo.genopole-toulouse.prd.fr/multalin/multalin.html]

96. Corpet F: Multiple sequence alignment with hierarchical clustering. Nucleic Acids Res 1988, 16:10881-10890.

97. Tamura K, Peterson D, Peterson N, Stecher G, Nei M, Kumar S: MEGA5: Molecular Evolutionary Genetics Analysis using Maximum Likelihood, Evolutionary Distance, and Maximum Parsimony Methods. Mol Biol Evol 2011, (submitted).

98. Swofford DL: PAUP*. Phylogenetic Analysis Using Parsimony (*and Other Methods). Version 4. Sunderland, Massachusetts: Sinauer Associates; 2003.

99. Ronquist F, Huelsenbeck JP: MrBayes 3: Bayesian phylogenetic inference under mixed models. Bioinformatics 2003, 19:1572-1574.

100. Huelsenbeck JP, Ronquist F: MRBAYES: Bayesian inference of phylogenetic trees. Bioinformatics 2001, 17:754-755.

101. Saitou N, Nei M: The neighbor-joining method: a new method for reconstructing phylogenetic trees. Mol Biol Evol 1987, 4:406-425.

102. Torrence JD, Bothwell TH: Tissue Iron Stores. In Methods in haematology. Edited by: Cook JD. New York: Churchill Livingston Press; 1980:104-109, [JD C (Series Editor)

doi:10.1186/1471-2148-11-106

Cite this article as: Neves et al.: Natural history of SLC11 genes in vertebrates: tales from the fish world. BMC Evolutionary Biology 2011 11:106. 